Copyright

by

Min Naing Lwin

2013 
The Thesis Committee for Min Naing Lwin

Certifies that this is the approved version of the following thesis:

\title{
Time-domain Modeling and Validation of Overcurrent/Reclosing Relay Operation
}

\author{
APPROVED BY \\ Supervising Committee:
}

Supervisors:

Surya Santoso

Mircea Driga 


\title{
Time-domain Modeling and Validation of Overcurrent/Reclosing Relay Operation
}

\author{
by \\ Min Naing Lwin, B.S. \\ Thesis \\ Presented to the Faculty of the Graduate School of \\ The University of Texas at Austin \\ in Partial Fulfillment \\ of the Requirements \\ for the Degree of
}

Master of Science in Engineering

The University of Texas at Austin

August 2013 


\section{Dedication}

This work is dedicated to my parents. Everything I have ever accomplished is owed to their dedication and sacrifice raising me and my brother. This work is also dedicated to my wife Teresa for her love, support, and encouragement. 


\section{Acknowledgments}

First and foremost, I would like to thank and express my sincere gratitude to my advisor, Dr. Surya Santoso. I am grateful for the opportunities he has given me, his continuing support, and valuable input. It has been a pleasure to collaborate with him on this and other projects. I would also like to thank Dr. Mircea Driga for his guidance and kindly agreeing to read the draft of this work.

I would like thank Jules Campbell for his assistance and input with the relay hardware and testing. I also would like to thank Schweitzer Engineering Labs for supplying their equipment to the University of Texas on permanent loan.

Finally, I sincerely thank my wife Teresa for her continuing support and encouragement throughout my graduate studies. 


\title{
Time-domain Modeling and Validation of Overcurrent/Reclosing Relay Operation
}

\author{
Min Naing Lwin, M.S.E. \\ The University of Texas at Austin, 2013 \\ Supervisor: Surya Santoso
}

The primary goal of this work is to develop a PSCAD/EMTDC simulation model which can emulate the reclosing capabilities of an actual reclosing relay. The first part of this work will demonstrate the capabilities of a commercially available, microprocessor-based reclosing relay, the SEL-551c. Next, a computer simulation model of this relay's reclosing capability will be developed in PSCAD/EMTDC and validated. The performance of the model will be compared to the performance of the SEL-551c. Because it is impractical to test the relay operation under fault conditions in a real distribution system, fault characteristics will be determined in PSCAD. Utilizing a test system for the SEL relay, the accuracy of the PSCAD recloser model will be compared to the SEL-551c relay for similar fault scenarios. The validation is done by analyzing the data from the simulation and experiment. The results show that both the PSCAD recloser model and SEL-551c operate close to the expected theoretical values.

The primary contribution of this work is the development of a PSCAD recloser model and validation with a real world reclosing relay. In previous works where recloser analysis was done in PSCAD, such as [14], recloser operation was manually accomplished. However, the recloser model developed in this work allows the user to enter any standard TCC equation that may be programmed into an actual relay and achieve similar results. The model is useful when analyzing larger distribution systems with multiple reclosers. Additionally, validating the PSCAD recloser model with a real world device provides confidence that the simulations provide reasonable and meaningful results. 


\section{Contents}

Contents vii

List of Tables $\quad$ ix

List of Figures $\quad$ xi

\begin{tabular}{lll}
\hline Introduction & $\mathbf{1}$
\end{tabular}

1.1 Motivation and Objective . . . . . . . . . . . . . . 1

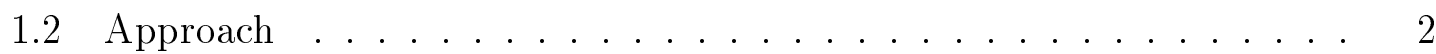

1.3 Results . . . . . . . . . . . . . . . . . . . . . . . . . . . . . . . . . . . .

1.4 Contribution . . . . . . . . . . . . . . . . . 3

2 Overview of Overcurrent Protection 4

3 Operation of a Typical Commercially Available Overcurrent/Reclosing Relay $\quad 8$

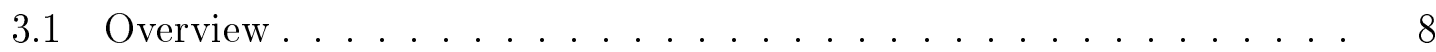

3.2 Instantaneous Overcurrent Elements . . . . . . . . . . . . . . . . . . 9

3.3 Time-Overcurrent Elements . . . . . . . . . . . . . . . . . . . . . . . . . . . . 12

3.4 Trip Logic . . . . . . . . . . . . . . . . . . . . . . . . 18

3.5 Reclosing Logic . . . . . . . . . . . . . . . . 20

3.5.1 Close Logic . . . . . . . . . . . . . . . 20

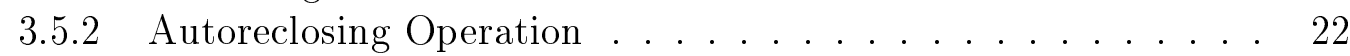

3.6 SEL Software . . . . . . . . . . . . . . . 24

4 Development of PSCAD/EMTDC Simulation Model 28

$4.1 \quad$ Distribution Circuit Model . . . . . . . . . . . . . . . . . . 29

4.2 Relay Model Development . . . . . . . . . . . . . . . . . 30

$4.2 .1 \quad$ Signal processing block . . . . . . . . . . . . . . . . . . . . . . 31

4.2 .2 TCC Curve Implementation . . . . . . . . . . . . . . . . . . . . . . . . . . . . 31

$4.2 .3 \quad$ Recloser Logic Implementation . . . . . . . . . . . . . . . . . . . 32

4.2 .4 Circuit Breaker Control . . . . . . . . . . . . . . 33

4.3 Simulation Results . . . . . . . . . . . . . . . . . . 34 
5 Reproducing Simulation Results in Hardware 36

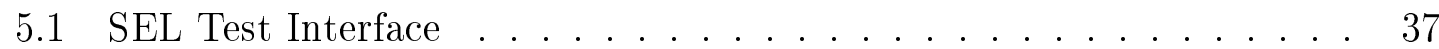

5.1 .1 Setting the Relay . . . . . . . . . . . . . . . . 37

5.1 .2 Creating Test Sequence in SEL 5401 . . . . . . . . . . . . 39

$5.1 .3 \quad$ Physical connections $\ldots \ldots \ldots \ldots \ldots$. . . . . . . . . . 41

5.2 Temporary Fault: One Fast Operation . . . . . . . . . . . . 42

5.3 Temporary Fault: Two Fast Operations . . . . . . . . . . . . . . . . 44

5.4 Permanent Fault: Two Fast Operations, Fuse Clears Fault . . . . . . 47

5.5 Permanent Fault: Two Fast, Two Delayed Operations and Recloser Lockout ......................... 49

$6 \quad$ Evaluating Performance of Recloser Model $\quad 51$

6.1 Overview . . . . . . . . . . . . . . . . . . . . . 51

6.2 Data Analysis . . . . . . . . . . . . . . . . . . . 52

6.3 Summary of Analysis . . . . . . . . . . . . . . . . 57

7 Conclusion and Future Work $\quad 59$

\begin{tabular}{ll}
\hline Bibliography & 61
\end{tabular} 


\section{List of Tables}

3.1 Summary of Instantaneous Overcurrent Elements and Pickup Settings . . . . . . . . . 10

3.2 Summary of Time-Overcurrent Elements and Pickup

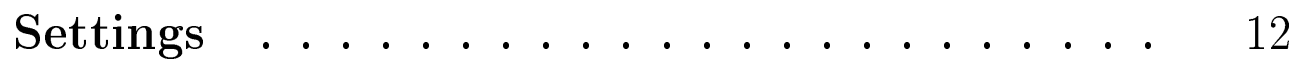

3.3 Available U.S. Curves [16] . . . . . . . . . . 17

$3.4 \quad$ Available I.E.C. Curves [16] . . . . . . . . . . 18

3.5 Elements Which Determine Relay Trip [16] . . 19

$3.6 \quad$ Elements Which Determine Relay Close . . . . 21

$4.1 \quad$ Desired PSCAD Circuit Breaker Operation . . 33

4.2 J-K Flip-Flop Truth Table Implementation . . . 34

4.3 Summary of Fault Scenarios Tested . . . . . . 34

$5.1 \quad$ Summary of SEL-551c Factory Settings . . . . 38

5.2 Summary of SEL-551c Settings For All Experi-

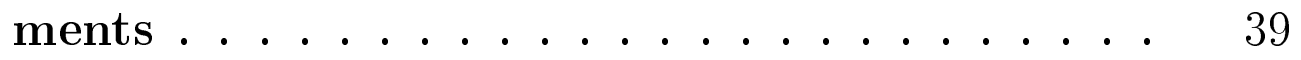

6.1 Theoretical Values From TCC Curves . . . . . . 53

6.2 Reclose Interval Settings . . . . . . . . . . 53

6.3 PSCAD Simulation Results For One Fast Oper-

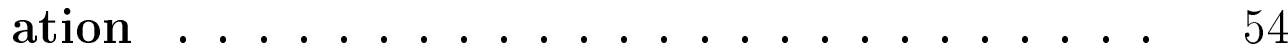

6.4 SEL-551c Results For One Fast Operation . . . 54

6.5 PSCAD Simulation Results for Two Fast Oper-

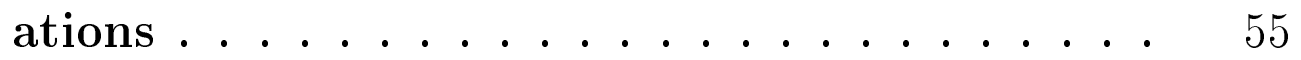

6.6 $\quad$ SEL-551c Results For Two Fast Operations . . . 55

6.7 PSCAD Simulation Results For Permanent Fault Downstream of Fuse . . . . . . . . . 56 
6.8 SEL-551c Results for Permanent Fault Downstream of Fuse . . . . . . . . . . . . . . . 56

6.9 PSCAD Simulation Results for Permanent Fault Upstream of Fuse . . . . . . . . . . . . . 57

6.10 SEL-551c Results for Permanent Fault Upstream of Fuse $\ldots \ldots \ldots \ldots \ldots \ldots$ 


\section{List of Figures}

2.0.1 An overview of protective relaying . . . . . 5

2.0.2 Example time current characteristic (TCC) curve

2.0.3 Development of overcurrent relays . . . . . . 7

3.1.1 Protective functions available for SEL-551c [16] 9

3.2.1 Phase instantaneous element 50P1 logic [16] . . 10

3.2.2 Single-phase instantaneous element 50A logic [16] 11

3.2.3 Neutral ground instantaneous element 50N1 logic

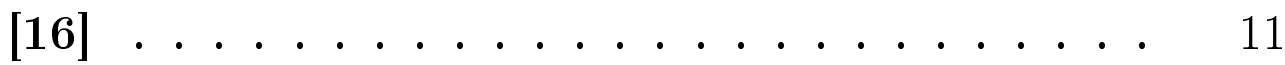

3.2.4 Residual ground instantaneous element 50G1 logic

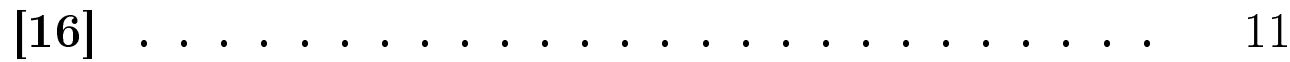

3.2.5 Negative-sequence instantaneous element 50Q1

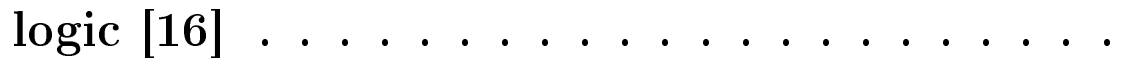

3.3.1 Phase time-overcurrent element 51P1T logic [16]

3.3.2 Neutral ground time-overcurrent element 51N1T

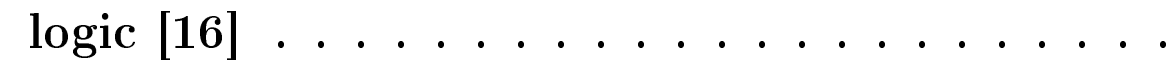

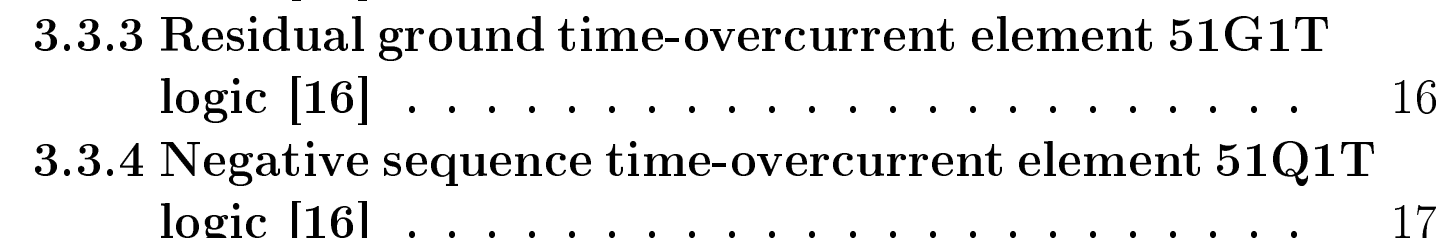

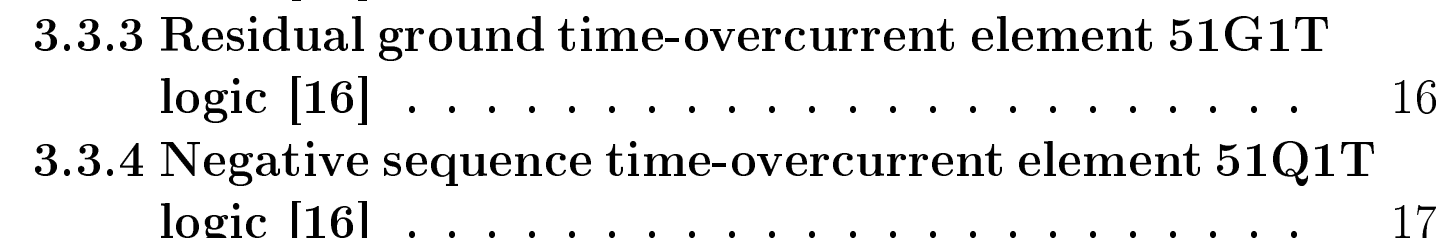

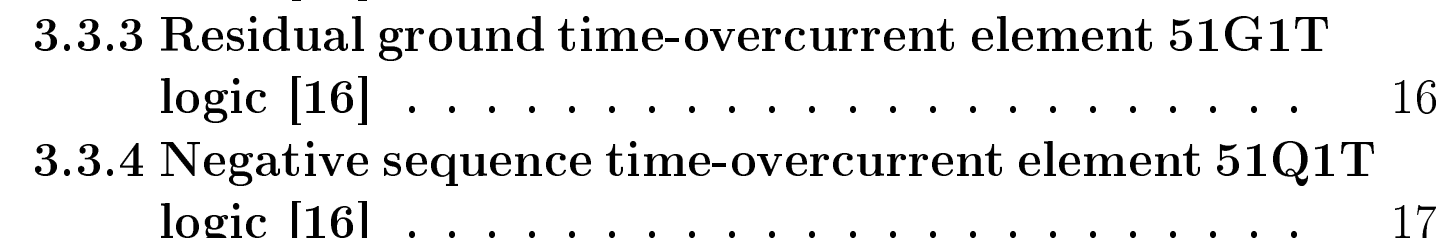

\begin{tabular}{|l|}
\hline 3.3.3 Residual ground time-overcurrent element 51G1T \\
\hline logic [16] . . . . . . . . . . . . . . 16 \\
\hline 3.3.4 Negative sequence time-overcurrent element 51Q1T \\
\hline logic [16] . . . . . . . . . . . . . . 17
\end{tabular} 14

3.4.1 Example SEL-551c trip operation [16] . . . . . 19

3.4.2 SEL-551c trip logic [16] . . . . . . . . . . 20

3.5.1 SEL-551c close logic [16] . . . . . . . . . . 21

3.5.2 Automatic reclosing logic [16] . . . . . . . 23 
3.6.1 Specifying SEL-551c relay settings using SEL AcSELerator QuickSet . . . . . . . . . 25

3.6.2 Simulating a single line-to-ground fault using SEL 5401................. 26

3.6.3 Event capture waveform from SEL-551c relay . 27

3.6.4 Event capture data from SEL-551c relay . . . . 27

4.1.1 One-line diagram of distribution circuit model 29

4.1.2 PSCAD distribution circuit model . . . . . . . 29

4.2.1 PSCAD reclosing relay model . . . . . . . . 30

4.3.1 Simulation results and corresponding reclosing sequences: a) one fast operation, b) two fast operations, c) two fast operations, fuse clears fault, d) two fast and two delayed operations, recloser lockout . . . . . . . . . . 35

5.1.1 Physical connections between SEL-551c and SEL

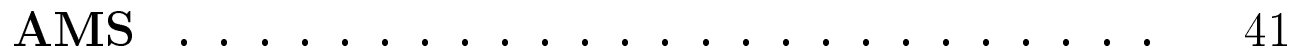

5.2.1 Expected recloser sequence for one fast opera-

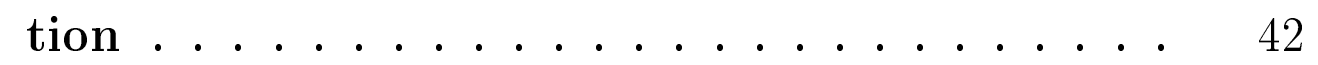

5.2.2 PSCAD simulation results for one fast operation 43

5.2.3 SEL event recorder data for one fast operation 44

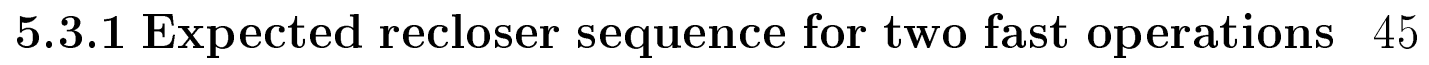

5.3.2 PSCAD simulation results for two fast operations 46

5.3.3 SEL event recorder data for two fast operations 46

5.4.1 Expected recloser sequence for permanent fault downstream of fuse . . . . . . . . . 47

5.4.2 PSCAD simulation results for permanent fault downstream of fuse . . . . . . . . . . 48

5.4.3 SEL event recorder data for permanent fault downstream of fuse . . . . . . . . . . . . 48

5.5.1 Expected recloser sequence for permanent fault upstream of fuse . . . . . . . . . . . 49

5.5.2 PSCAD results for permanent fault upstream of

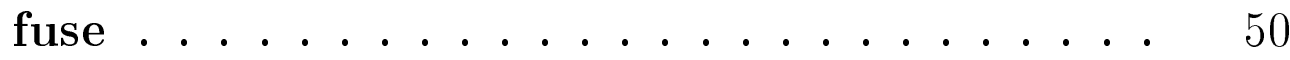


5.5.3 SEL event recorder data for permanent fault upstream of fuse . . . . . . . . . 50

6.1.1 Overview of data flow . . . . . . . . . 52 


\section{1}

\section{Introduction}

\subsection{Motivation and Objective}

With the improvements in technology and reduced cost, Intelligent Electronic Devices (IEDs) have been commonly incorporated into the electric power grid over the past decade. These devices include devices that are capable of capturing, storing, and transmitting voltage and current waveform data. The IED of particular interest in this work is the autoreclosing relay, also known as an autorecloser or recloser. Because most faults in the distribution system are temporary, the purpose of a recloser is to similar to an overcurrent relay; however, it is capable of attempting to energize a faulted circuit multiple times after a programmed time delay in order to prevent unnecessary loss of service during a temporary fault.

The primary goal of this work is to develop a PSCAD/EMTDC simulation model which can emulate the reclosing capabilities of an actual reclosing relay. The first part of this work will demonstrate the capabilities of a commercially available, microprocessor-based reclosing relay, the SEL-551c. Next, a computer simulation model of this relay's reclosing capability will be developed in PSCAD/EMTDC and validated. The performance of the model will be compared to the performance of the SEL-551c. Because it is impractical to test the relay operation under fault conditions in a real distribution system, fault characteristics will be determined in PSCAD. Utilizing a test system for the SEL relay, the accuracy of the PSCAD recloser model will be compared to the SEL-551c relay for similar fault scenarios. The validation is done by analyzing the data from the simulation and experiment. 


\subsection{Approach}

The approach of this work is to first cover the background on overcurrent protection and reclosing and then proceed with the development of the PSCAD simulation model and SEL-551c experiments. To achieve this, Chapter 2 will first briefly provide some background on overcurrent protection. Next, the capabilities and operation of the SEL-551c relay will be discussed in Chapter 3 to provide an understanding of the test methodology discussed in later chapters. Chapter 3 will cover the relay's overcurrent elements, trip and reclosing logic, event capture capabilities, and associated software. Chapter 4 will then discuss the development of the PSCAD recloser model. This discussion will include the development of the recloser logic using available PSCAD modules and provide initial test results. Four single line-to-ground fault scenarios are tested, each resulting in different reclosing sequences. These reclosing sequences are: one fast operation, two fast operations, two fast operations with fault cleared by fuse, and two fast and two delayed operations with recloser lockout. These different sequences are achieved by placing the fault in different locations and varying the fault duration. Chapter 5 will then detail how the data from the PSCAD simulations is used to develop a experiments to test the operation of the SEL-551c under the same fault conditions. The experiments will involve utilizing the SEL test interface and SEL-AMS signal generator. Data will then be collected from the relay's event capture capability and compared to the PSCAD simulation. The parameters of interest are the fault current duration or relay operating time and the reclose interval duration. Using these parameters, one can determine if the SEL-551c relay and PSCAD recloser model operate accurately under the specified conditions.

\subsection{Results}

The results for each of the fault scenarios are presented in Chapter 6. First, the theoretical relay operating times and reclose interval durations are determined by using the specified TCC curves and relay settings. Next, the same relay operating times and reclose interval durations are determined from the PSCAD simulation waveforms by manual calculation. Finally, the corresponding SEL-551c values are determined using a combination of event capture data and relay I/O signal measurements. The 
results show that both the PSCAD recloser model and SEL-551c operate close to the expected theoretical values.

\subsection{Contribution}

The primary contribution of this work is the development of a PSCAD recloser model and validation with a real world reclosing relay. In previous works where recloser analysis was done in PSCAD, such as [14], recloser operation was manually accomplished. That is, the circuit breaker trip and close operations were controlled by preset time delays. However, the recloser model developed in this work allows the user to enter any standard TCC equation that may be programmed into an actual relay and achieve similar results. The model becomes more useful when analyzing larger distribution systems with multiple reclosers. Multiple recloser blocks developed in this work can be inserted into a complex distribution circuit model without the need to manually calculate and program successive time delays for each circuit breaker. Additionally, the operating performance comparison with a commercial microprocessor-based reclosing relay is unique. Validating the PSCAD recloser model with a real world device provides confidence that the simulations provide reasonable and meaningful results. Moreover, the methodology developed in this work can be applied to the modeling and validation of other relays. 


\section{2}

\section{Overview of Overcurrent Protection}

Overcurrent protection is a basic yet important component of any power system. When a fault occurs on a distribution system, typically a circuit breaker or fuse must be opened to isolate the faulted section. The circuit breaker, however, requires another device, a relay, to provide the signal of when to open or close. Therefore, the primary function of a relay is to disconnect the associated device (distribution line, generator, transformer, bus, etc.) from the power system by opening a circuit breaker when an unwanted condition is detected, typically as a result of a fault. The relay will detect faults and abnormal operating conditions and then initiate an action as quickly as possible to return the power system back to its normal state. This action helps maintain as much of the power system as possible in steady state. Note that the goal is not necessarily to entirely prevent damage to the system. In fact, relays will only operate after some small yet detectable stress has occurred and attempt to limit further stress on equipment. Therefore, the speed of operation is a key requirement resulting in typical response times of overcurrent protective devices on the order of a few cycles. Because human intervention is not possible at these speeds, the overcurrent protection system must be automated [17].

A general overview of protective relaying is provided in Figure 2.0.1. The state of the power system is observed by the relay through current transformers (CTs) and potential transformers (PTs). In a microprocessor-based relay, this input is sent to the signal processing unit which converts the analog signal into digital. The CPU in the relay then determines if an unwanted condition exists on the system. There can be several protective functions provided by the CPU including instantaneous overcurrent and time-overcurrent functions. If the appropriate protective elements 
indicate that a problem exists and satisfy the relay's trip logic equation, the relay will provide a signal to the circuit breaker to open. Similarly, a close signal may be sent to the circuit breaker if the appropriate conditions are met. Therefore, there are three key protective requirements of protective relaying: diagnosing the problem correctly, initiating appropriate corrective action, and operating the protective devices that cause the least disturbance to the power system.

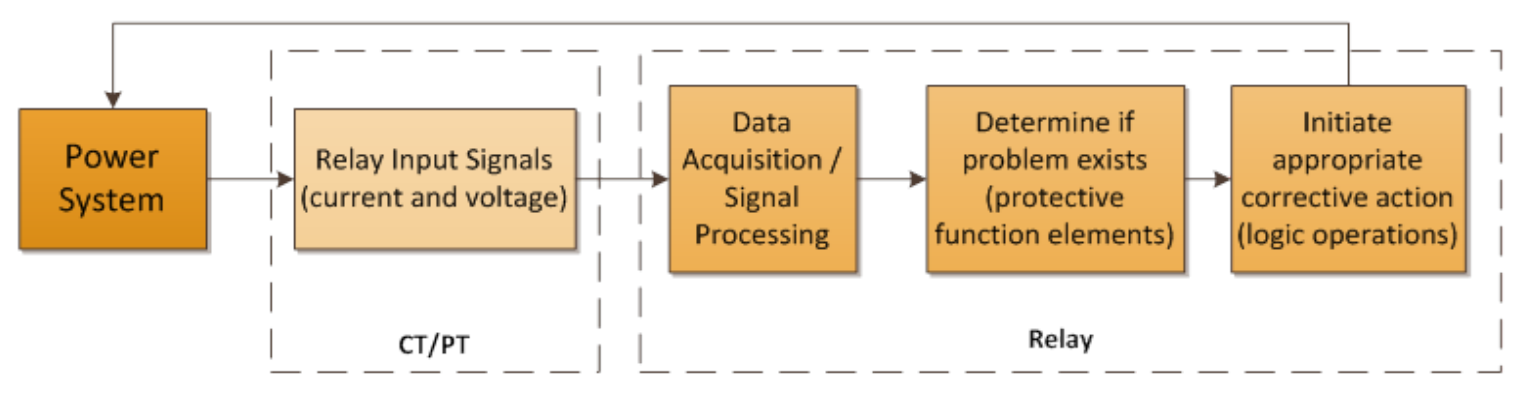

Figure 2.0.1: An overview of protective relaying

Furthermore, the topology of the power system will impact which method of protection is deployed. This work will only consider distribution systems which are typically radial. This means that the system can be modeled as one equivalent source with multiple loads and branches extending radially from the source. When a protective device operates, e.g. a circuit breaker, recloser, or fuse, all customers downstream on the system will be disconnected. Therefore, it is imperative to minimize the section of the power system which is disconnected while isolating the fault. This typically means that the protective device closest and upstream of the fault must be disconnected. However, the fault current on a radial distribution system will be seen by all devices upstream of the fault. In order to coordinate many devices in a radial system, time delays are introduced for protective devices further upstream. That means that protective devices at the end of a radial distribution system operate much faster than those closer to the source. Due to this intentional time delay, overcurrent protective devices are able to be coordinated through this inverse-time characteristic. This inverse-time characteristic is mathematically defined on a graph known as a time-current characteristic (TCC) curve. An example of possible TCC 
settings for a relay is shown in Figure 2.0.2 [16]. The curves specify the time delay before the relay will operate for a given multiple of the pickup current setting. For example, as shown in the figure, if the fault current is 4 times the pickup setting, the relay will trip around 0.04 seconds. Multiple curves can be set for a reclosing relay to operate in fuse saving mode.

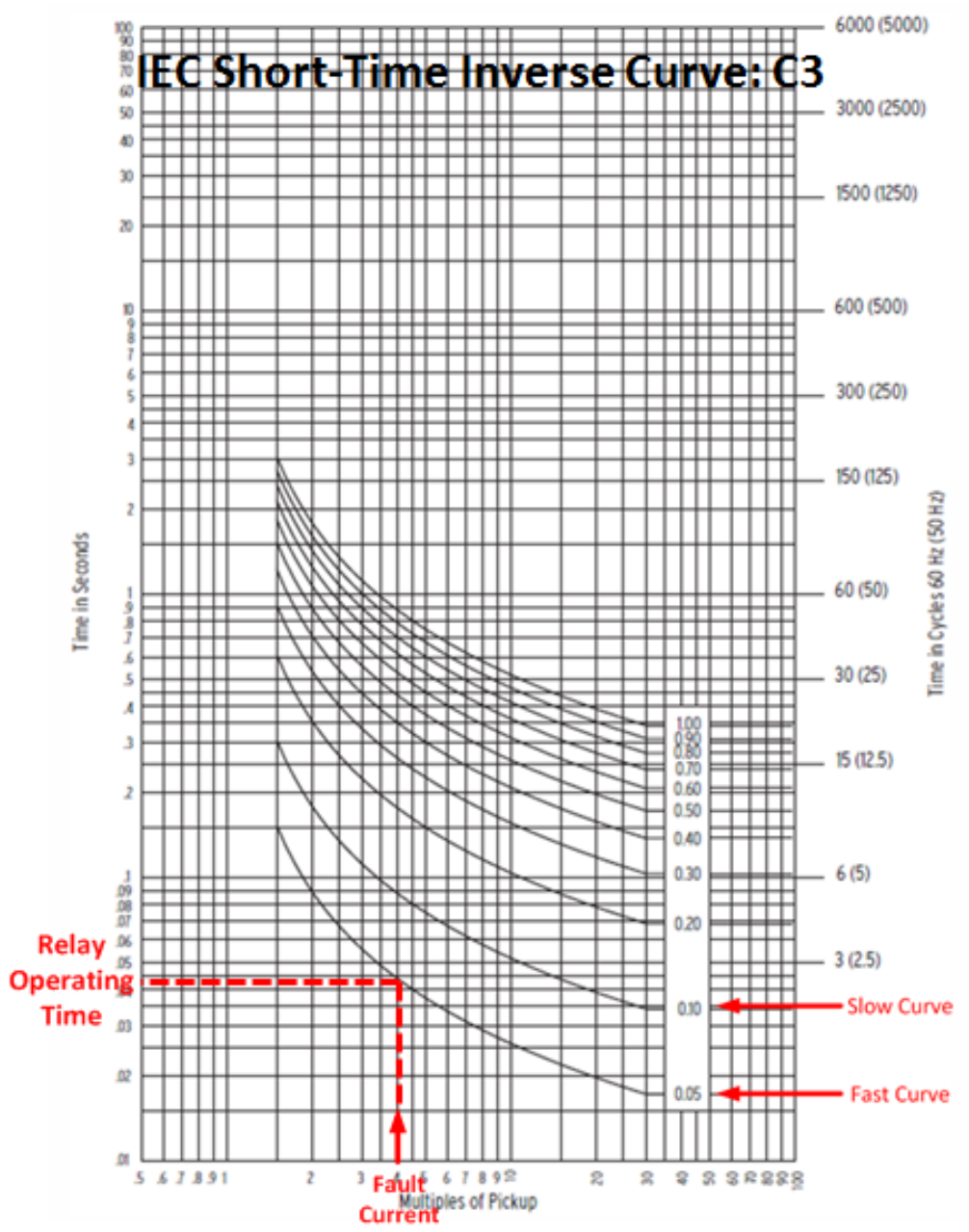

Figure 2.0.2: Example time current characteristic (TCC) curve

Although relays and inverse time-overcurrent protective devices have been utilized since the early days of the power system, recent advances in technology have made 
microprocessor-based relays increasingly popular. Figure 2.0.3 shows a time-line of the progression of technology. The first time-overcurrent relays were electromechanical and now most modern overcurrent relays incorporate some kind of microprocessor. One major advantage is the incorporation of multiple protective functions into one device. Additionally, the relay can have remote control and monitoring capabilities depending on available communications. Furthermore, event recorders in the relay can capture waveforms of abnormal events, such as faults, providing valuable data. The SEL-551c is such a relay and will be discussed in the following chapter.

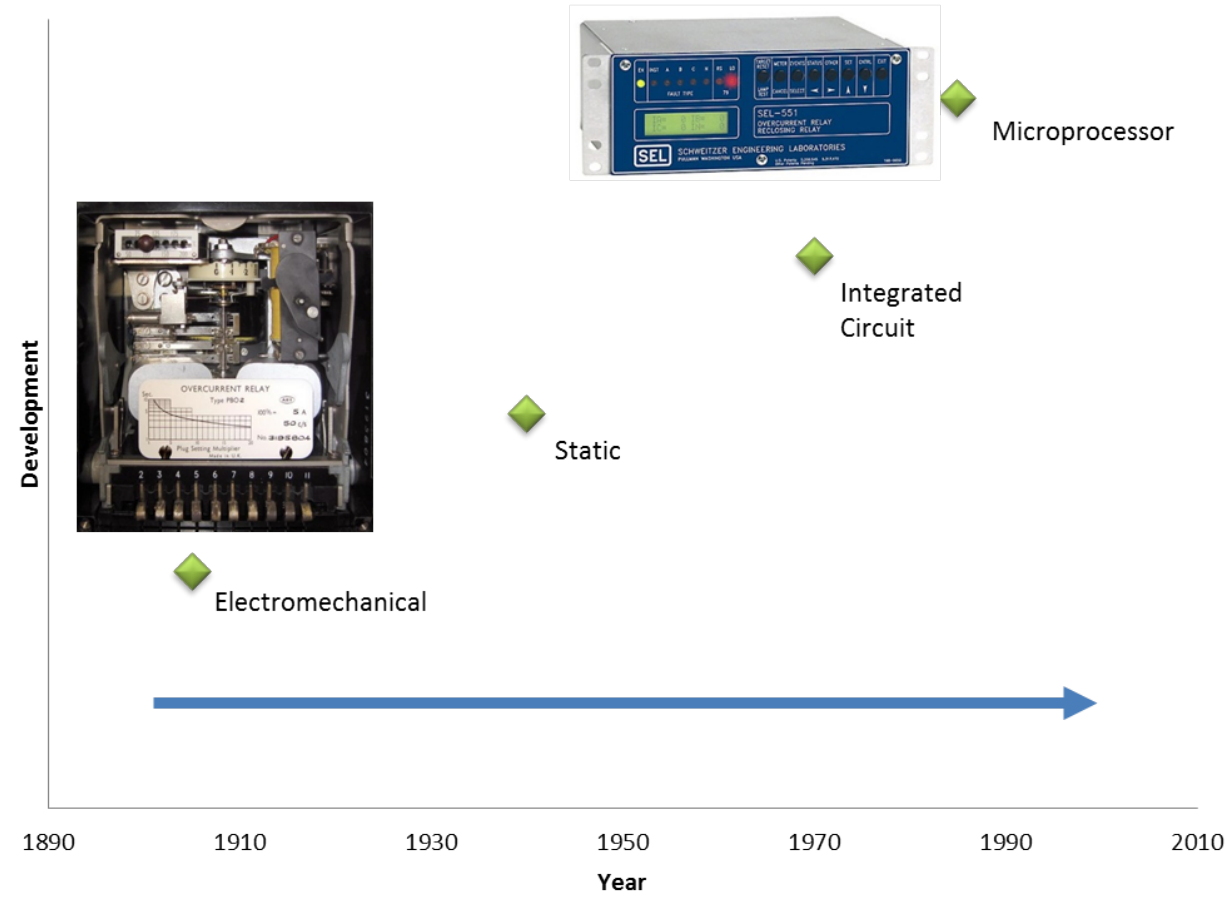

Figure 2.0.3: Development of overcurrent relays 


\section{3 \\ Operation of a Typical Commercially Available Overcurrent/Reclosing Relay}

\subsection{Overview}

The purpose of this chapter is to describe the methodology for setting and implementing an overcurrent/reclosing relay such as the SEL-551c. To accomplish this objective, the chapter first describes the available functions in the relay, then explains the trip, close, and reclosing logic, and finally summarizes the software required to interface with the relay. The SEL-551C is a microprocessor-based overcurrent/reclosing relay. This means that it provides the functions of overcurrent protection and up to four shots of reclosing. The relay measures line and neutral currents only and does not measure voltage. Because it is microprocessor based, line and neutral current inputs are sampled at evenly spaced intervals, in this case, 8 samples per cycle. Additional features include optoisolated inputs, output contacts, event recorder reports stored in non-volatile memory, multiple overcurrent and time-overcurrent elements, programmable logic equations, and demand ammetering [16]. Figure 3.1.1 shows a schematic using IEEE standard device numbers to illustrate the SEL-551c capabilities.

The logic for the trip, close, and reclose functions operate based on the status of variables (relay word bits) in the relay firmware, referred to as elements. The relay includes several elements for each of the desired overcurrent and reclosing functions. These elements and their corresponding settings are detailed in the following sections. 
Note that element and setting names are selected by the manufacturer and cannot be changed.

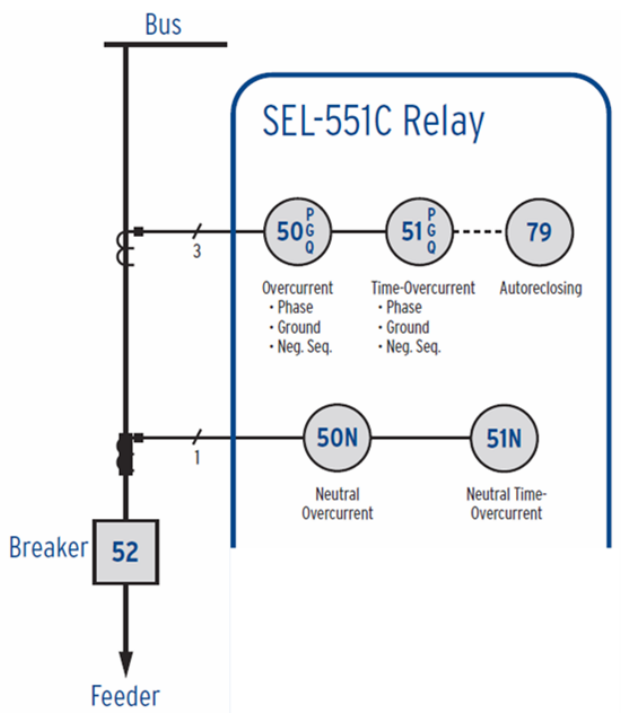

Figure 3.1.1: Protective functions available for SEL-551c [16]

\subsection{Instantaneous Overcurrent Elements}

The basic overcurrent function of the SEL-551c relay is instantaneous overcurrent protection. For this function, the relay will operate without any intentional time delay if the current exceeds a preset threshold or pickup setting. The five instantaneous overcurrent functions are phase, single-phase, neutral ground, residual ground, and negative-sequence. 
Table 3.1: Summary of Instantaneous Overcurrent Elements and Pickup Settings

\begin{tabular}{|c|c|c|c|c|}
\hline Function & No. of Elements & No. of Settings & Element Name(s) & Setting Name(s) \\
\hline \hline Phase & 6 & 6 & $50 \mathrm{P} 1-50 \mathrm{P} 6$ & $50 \mathrm{P} 1 \mathrm{P}-50 \mathrm{P} 6 \mathrm{P}$ \\
\hline Single-Phase & 3 & 1 & $50 \mathrm{~A}, 50 \mathrm{~B}, 50 \mathrm{C}$ & $50 \mathrm{ABCP}$ \\
\hline Neutral Ground & 2 & 2 & $50 \mathrm{~N} 1,50 \mathrm{~N} 2$ & $50 \mathrm{~N} 1 \mathrm{P}, 50 \mathrm{~N} 2 \mathrm{P}$ \\
\hline Residual Ground & 2 & 2 & $50 \mathrm{G} 1,50 \mathrm{G} 2$ & $50 \mathrm{G} 1 \mathrm{P}, 50 \mathrm{G} 2 \mathrm{P}$ \\
\hline Negative-Sequence & 2 & 2 & $50 \mathrm{Q} 1,50 \mathrm{Q} 2$ & $50 \mathrm{Q} 1 \mathrm{P}, 50 \mathrm{Q} 2 \mathrm{P}$ \\
\hline
\end{tabular}

The SEL-551c includes six different phase instantaneous overcurrent elements and six corresponding settings, shown in Table 3.1. This function operates by comparing the maximum phase current $I_{P}$ (where $I_{P}=\max$ of $I_{A}, I_{B}$, or $I_{C}$ ) with the specified pickup setting for each element. If $I_{P}$ exceeds any of the six specified pickup settings, the corresponding element will change to a logical 1. For example, following the logic of Figure 3.2.1, if $I_{P}$ exceeds the phase instantaneous overcurrent pickup setting $50 \mathrm{P} 1 \mathrm{P}$, i.e., $I_{P}>50 \mathrm{P} 1 \mathrm{P}$, then relay word bit 50P1 will be set to logical 1 (50P1 $=$ logical 1). The operation for 50P2-50P6 is similar with corresponding pickup settings 50P2P - 50P6P. Note that any of these elements can be disabled using the SEL software interface.

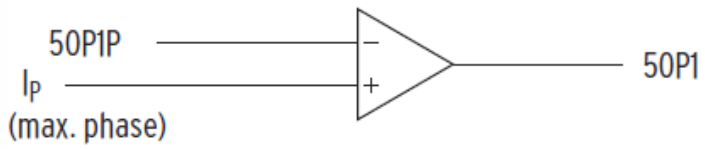

Figure 3.2.1: Phase instantaneous element 50P1 logic [16]

The SEL-551c includes three different single-phase instantaneous elements and one corresponding pickup setting shown in Table 3.1. This function operates by comparing the magnitude of each single-phase current $I_{A}, I_{B}$, or $I_{C}$ with the pickup setting, 50ABCP. If any of the single-phase currents exceed the specified pickup setting, the corresponding element will change to a logical 1. For example, following the logic of Figure 3.2 .2 , if $I_{A}$ exceeds the pickup setting 50ABCP, i.e., $I_{A}>50 \mathrm{ABCP}$, then the relay word bit $50 \mathrm{~A}$ will be set to logical $1(50 \mathrm{~A}=$ logical 1$)$. Elements $50 \mathrm{~B}$ and 50C operate similarly. Note that any of these elements can be disabled using the SEL software interface. 


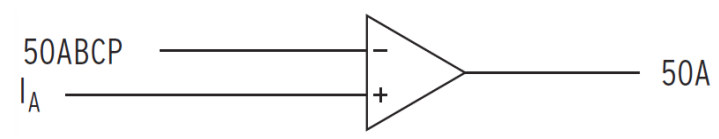

Figure 3.2.2: Single-phase instantaneous element 50A logic [16]

The SEL-551c includes two different neutral ground instantaneous elements and two corresponding pickup settings shown in Table 3.1. This function operates by comparing the magnitude of the neutral ground current $I_{N}$ with the specified pickup setting for each element. $I_{N}$ is a hardwired input to the relay. If $I_{N}$ exceeds any of the specified pickup settings, the corresponding element will change to a logical 1. For example, following the logic of Figure 3.2.3. if $I_{N}$ exceeds the pickup setting $50 \mathrm{~N} 1 \mathrm{P}$, i.e., $I_{N}>50 \mathrm{~N} 1 \mathrm{P}$, then the relay word bit $50 \mathrm{~N} 1$ will be set to logical 1 (50N1 $=$ logical 1). Element 50N2 operates similarly. Note that any of these elements can be disabled using the SEL software interface.

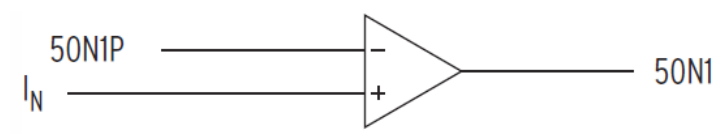

Figure 3.2.3: Neutral ground instantaneous element 50N1 logic [16]

The SEL-551c includes two different residual ground instantaneous elements and two corresponding pickup settings shown in Table 3.1. This function operates by comparing the magnitude of the residual ground current $I_{G}$ (where $I_{G}$ is derived within the SEL-551c firmware using $I_{A}, I_{B}$, and $I_{C}$ ) with the specified pickup setting for each element. If $I_{G}$ exceeds any of the specified pickup settings, the corresponding element will change to a logical 1. For example, following the logic of Figure 3.2.4, if $I_{G}$ exceeds the pickup setting $50 \mathrm{G} 1 \mathrm{P}$, i.e., $I_{G}>50 \mathrm{G} 1 \mathrm{P}$, then the relay word bit $50 \mathrm{G} 1$ will be set to logical $1(50 \mathrm{G} 1=$ logical 1$)$. Element 50G2 operates similarly. Note that any of these elements can be disabled using the SEL software interface.

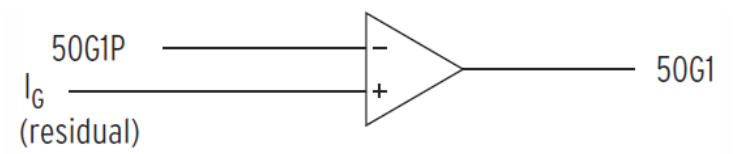

Figure 3.2.4: Residual ground instantaneous element 50G1 logic [16] 
The SEL-551c includes two different negative-sequence ground instantaneous elements and two corresponding pickup settings shown in Table 3.1. This function operates by comparing the magnitude of the negative-sequence current $3 I_{2}$ (where $3 I_{2}$ is derived within the SEL-551c firmware using $I_{A}, I_{B}$, and $I_{C}$ ) with the specified pickup setting for each element. If $3 I_{2}$ exceeds any of the specified pickup settings, the corresponding element will change to a logical 1. For example, following the logic of Figure 3.2.5, if $3 I_{2}$ exceeds the pickup setting 50Q1P, i.e., $3 I_{2}>50 \mathrm{Q} 1 \mathrm{P}$, then the relay word bit 50Q1 will be set to logical 1 (50Q1 = logical 1). Element 50Q2 operates similarly. Note that any of these elements can be disabled using the SEL software interface [16].

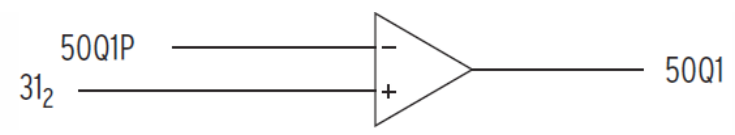

Figure 3.2.5: Negative-sequence instantaneous element 50Q1 logic [16]

\subsection{Time-Overcurrent Elements}

Of particular interest in this work, are the inverse time-overcurrent functions of the SEL-551c relay. For this protective function, the relay will operate with an intentional time delay inversely proportional to the measured current. In other words, if the current exceeds a preset threshold or pickup setting, the operating time of the relay is smaller for increasing values of fault current. For the SEL-551c, the operating time is defined analytically as a function of multiples of pickup current and timedelay setting. There are ten such curves available. These equations are provided in the following section. The four time-overcurrent functions provided by the SEL-551c relay are phase, neutral ground, residual ground, and negative-sequence.

Table 3.2: Summary of Time-Overcurrent Elements and Pickup Settings

\begin{tabular}{|c|c|c|}
\hline Time-Overcurrent Function & Element(s) & Pickup \\
\hline \hline Phase & 51P1T, 51P2T & 51P1P, 51P2P \\
\hline Neutral Ground & $51 \mathrm{~N} 1 \mathrm{~T}$ & $51 \mathrm{~N} 1 \mathrm{P}$ \\
\hline Residual Ground & $51 \mathrm{G} 1 \mathrm{~T}$ & $51 \mathrm{G} 1 \mathrm{P}$ \\
\hline Negative-Sequence & $51 \mathrm{Q} 1 \mathrm{~T}, 51 \mathrm{Q} 2 \mathrm{~T}$ & $51 \mathrm{Q} 1 \mathrm{P}$, 51Q2P \\
\hline
\end{tabular}


The SEL-551c includes two phase time-overcurrent elements and two corresponding settings, shown in Table 3.2. This function operates by comparing the maximum phase current $I_{P}$ (where $I_{P}=\max$ of $I_{A}, I_{B}$, or $I_{C}$ ) with the specified pickup setting for each element. Note that this input is controlled by the Torque Control Switch which allows the input to be enabled or disabled from an external output. For the first phase element, 51P1T, additional settings include 51P1C (curve type), 51P1TD (time dial setting), and 51P1RS (electromechanical reset). 51P1RS allows the SEL$551 \mathrm{c}$ to emulate the reset timing of an electromechanical relay. For the purposes of this work, 51P1RS will be disabled. The second phase element, 51P2T, operates similarly with 51P2RS also disabled.

If $I_{P}$ exceeds the specified pickup settings, the corresponding element will begin the timer. As long as the measured current $I_{P}$ exceeds the pickup setting, the timer will continue until it times out based on the specified curve type and time dial setting. At this point, the curve timeout indication will change to a logical 1. For example, following the logic of Figure 3.3.1, if $I_{P}$ exceeds the pickup setting 51P1P, i.e., $I_{P}>$ $51 \mathrm{P} 1 \mathrm{P}$, then the timer will begin based on 51P1C and 51P1TD. If the overcurrent condition persists long enough, relay word bit 51P1T will be set to logical 1 (51P1T $=$ logical 1 ). The operation for $51 \mathrm{P} 2 \mathrm{~T}$ is similar with corresponding pickup settings 51P2P. Note that any of these elements can be disabled using the SEL software interface. Complete detail on the operation of the time-overcurrent elements can be found in [16]. 


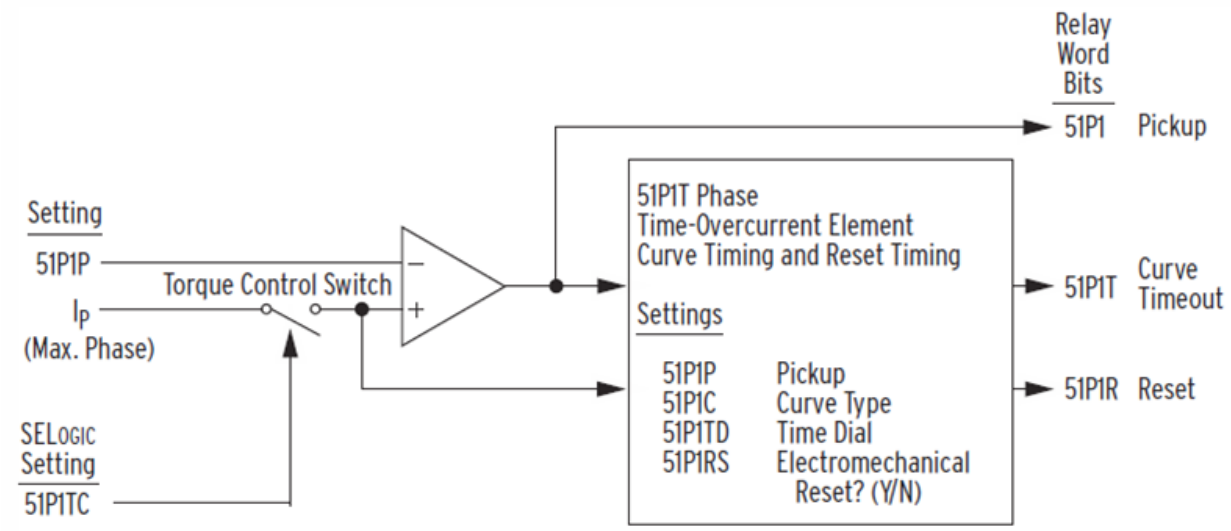

SELOGIC Setting 51P1TC Controls the Torque Control Switch

\begin{tabular}{l|c} 
51P1TC State & $\begin{array}{c}\text { Torque Control } \\
\text { Switch Position }\end{array}$ \\
\hline Logical 1 & Closed \\
Logical 0 & Open
\end{tabular}

\begin{tabular}{c|l}
$\begin{array}{l}\text { Setting } \\
\text { 51P1RS }=\end{array}$ & Reset Timing \\
\hline Y & Electromechanical \\
N & 1 Cycle
\end{tabular}

Figure 3.3.1: Phase time-overcurrent element 51P1T logic [16]

The SEL-551c includes one neutral ground time-overcurrent element and corresponding settings, shown in Table 3.2. This function operates by comparing the maximum phase current $I_{P}$ (where $I_{P}=\max$ of $I_{A}, I_{B}$, or $I_{C}$ ) with the specified pickup setting. Note that this input is also controlled by a Torque Control Switch which allows the input to be enabled or disabled from an external output. Similar to the phase time-overcurrent element, additional settings include 51N1C (curve type), 51N1TD (time dial setting), and 51N1RS (electromechanical reset). For the purposes of this work, 51N1RS will be disabled. 


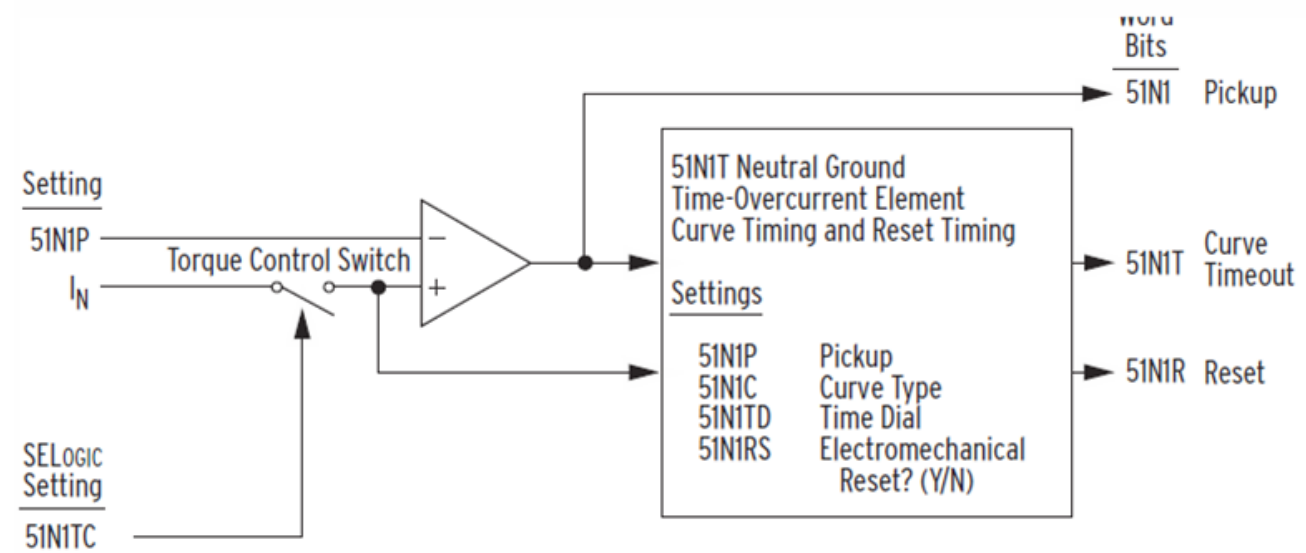

SELOGIC Setting 51N1TC Controls the Torque Control Switch

\begin{tabular}{l|c} 
51N1TC State & $\begin{array}{c}\text { Torque Control } \\
\text { Switch Position }\end{array}$ \\
\hline Logical 1 & $\begin{array}{l}\text { Closed } \\
\text { Open }\end{array}$
\end{tabular}

\begin{tabular}{c|l}
$\begin{array}{l}\text { Setting } \\
\text { 51N1RS }=\end{array}$ & Reset Timing \\
\hline Y & $\begin{array}{l}\text { Electromechanical } \\
\text { N Cycle }\end{array}$
\end{tabular}

Figure 3.3.2: Neutral ground time-overcurrent element 51N1T logic [16]

The SEL-551c includes one residual ground time-overcurrent element and corresponding settings, shown in Table 3.2. This function operates by comparing the maximum phase current $I_{P}$ (where $I_{P}=\max$ of $I_{A}, I_{B}$, or $I_{C}$ ) with the specified pickup setting. Note that this input is also controlled by a Torque Control Switch which allows the input to be enabled or disabled from an external output. Similar to the phase time-overcurrent element, additional settings include 51G1C (curve type), 51G1TD (time dial setting), and 51G1RS (electromechanical reset). For the purposes of this work, 51G1RS will be disabled. 


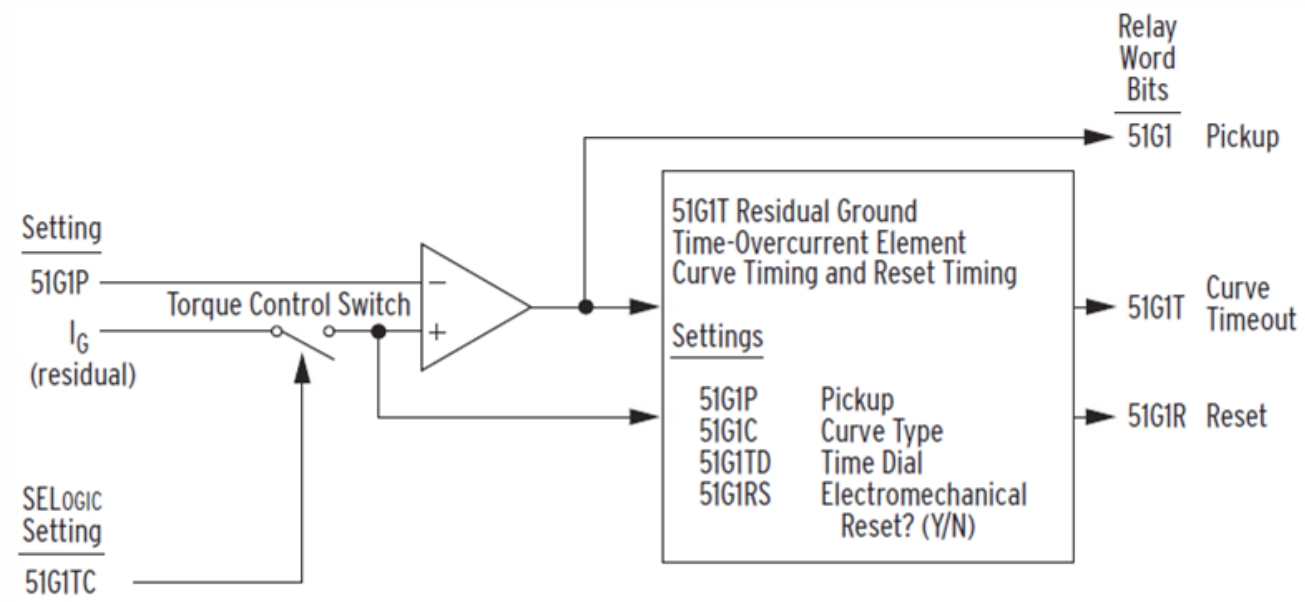

SELOGIC Setting 51G1TC Controls the Torque Control Switch

\begin{tabular}{l|c} 
51G1TC State & $\begin{array}{c}\text { Torque Control } \\
\text { Switch Position }\end{array}$ \\
\hline Logical 1 & $\begin{array}{l}\text { Closed } \\
\text { Open }\end{array}$
\end{tabular}

\begin{tabular}{c|l}
$\begin{array}{l}\text { Setting } \\
\text { 51G1RS }=\end{array}$ & Reset Timing \\
\hline Y & $\begin{array}{l}\text { Electromechanical } \\
\text { N Cycle }\end{array}$
\end{tabular}

Figure 3.3.3: Residual ground time-overcurrent element 51G1T logic [16]

Lastly, the SEL-551c includes two negative sequence time-overcurrent elements and two corresponding settings, shown in Table 3.2. This function operates by comparing the maximum phase current $I_{P}$ (where $I_{P}=\max$ of $I_{A}, I_{B}$, or $I_{C}$ ) with the specified pickup setting for each element. Note that this input is controlled by the Torque Control Switch which allows the input to be enabled or disabled from an external output. For the first phase element, 51Q1T, additional settings include 51Q1C (curve type), 51Q1TD (time dial setting), and 51Q1RS (electromechanical reset). 51Q1RS allows the SEL-551c to emulate the reset timing of an electromechanical relay. For the purposes of this work, 51Q1RS will be disabled. The second phase element, 51Q2T, operates similarly with 51PQ2RS also disabled. 


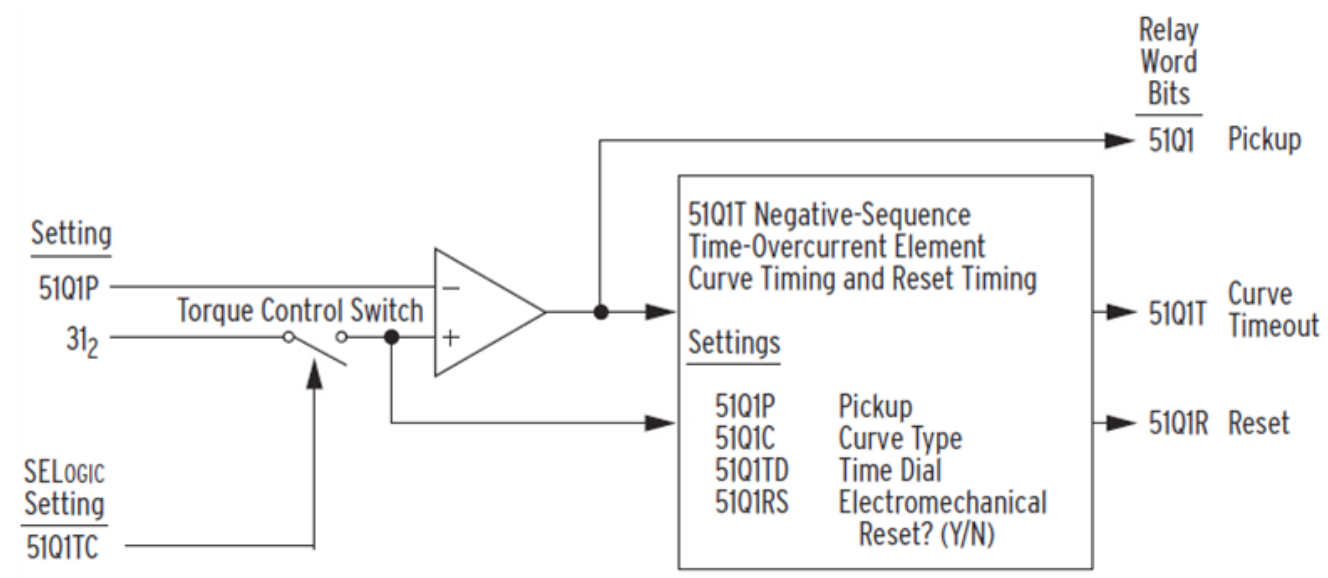

SELOGIC Setting 5101TC Controls the Torque Control Switch

\begin{tabular}{l|c} 
5101TC State & $\begin{array}{c}\text { Torque Control } \\
\text { Switch Position }\end{array}$ \\
\hline Logical 1 & $\begin{array}{c}\text { Closed } \\
\text { Open }\end{array}$
\end{tabular}

\begin{tabular}{|c|c|}
\hline $\begin{array}{l}\text { Setting } \\
\text { 5101RS }=\end{array}$ & Reset Timing \\
\hline $\begin{array}{l}Y \\
N\end{array}$ & $\begin{array}{l}\text { Electromechanical } \\
1 \text { Cycle }\end{array}$ \\
\hline
\end{tabular}

Figure 3.3.4: Negative sequence time-overcurrent element 51Q1T logic [16]

There are ten curve types available or each of the time-overcurrent elements. The curves and their associated equations are listed in Tables 3.3 and 3.4 . Each equation specifies the operating time (seconds) $t_{p}$, as a function of the time dial setting, TD, and the applied multiples of pickup current, $\mathrm{M}$.

Table 3.3: Available U.S. Curves [16]

\begin{tabular}{|c|c|}
\hline Curve Type & Equation \\
\hline \hline Moderately Inverse (U1) & $\mathrm{t}_{\mathrm{p}}=\mathrm{TD} \cdot\left(0.0226+\frac{0.0104}{\mathrm{M}^{0} .02-1}\right)$ \\
\hline Inverse (U2) & $\mathrm{t}_{\mathrm{p}}=\mathrm{TD} \cdot\left(0.180+\frac{5.95}{\mathrm{M}^{2}-1}\right)$ \\
\hline Very Inverse (U3) & $\mathrm{t}_{\mathrm{p}}=\mathrm{TD} \cdot\left(0.0963+\frac{3.88}{\mathrm{M}^{2}-1}\right)$ \\
\hline Extremely Inverse (U4) & $\mathrm{t}_{\mathrm{p}}=\mathrm{TD} \cdot\left(0.0352+\frac{5.67}{\mathrm{M}^{2}-1}\right)$ \\
\hline Short-Time Inverse (U5) & $\mathrm{t}_{\mathrm{p}}=\mathrm{TD} \cdot\left(0.00262+\frac{0.0342}{\mathrm{M}^{0.002}-1}\right)$ \\
\hline
\end{tabular}

Note: Allowed time dial settings are between 0.5 and 15. 
Table 3.4: Available I.E.C. Curves [16]

\begin{tabular}{|c|c|}
\hline Curve Type & Equation \\
\hline \hline Class A, Standard Inverse (C1) & $\mathrm{t}_{\mathrm{p}}=\mathrm{TD} \cdot\left(\frac{0.14}{\mathrm{M}^{0.02-1}}\right)$ \\
\hline Class B, Very Inverse (C2) & $\mathrm{t}_{\mathrm{p}}=\mathrm{TD} \cdot\left(\frac{13.5}{\mathrm{M}-1}\right)$ \\
\hline Class C, Extremely Inverse (C3) & $\mathrm{t}_{\mathrm{p}}=\mathrm{TD} \cdot\left(\frac{80}{\mathrm{M}^{2}-1}\right)$ \\
\hline Long-Time Inverse (C4) & $\mathrm{t}_{\mathrm{p}}=\mathrm{TD} \cdot\left(\frac{120}{\mathrm{M}-1}\right)$ \\
\hline Short-Time Inverse $(\mathrm{C} 5)$ & $\mathrm{t}_{\mathrm{p}}=\mathrm{TD} \cdot\left(\frac{0.05}{\mathrm{M}^{0.04}-1}\right)$ \\
\hline
\end{tabular}

Note: Allowed time dial settings are between 0.05 and 1.

\subsection{Trip Logic}

In any reclosing sequence, the first action is to trip the relay after an overcurrent condition is sensed. Accordingly, for the SEL-551c to perform a reclose operation, it must first sense the overcurrent condition and make the decision to output a trip signal. This section will cover the operation of the SEL-551c trip logic. Any one or combination of the instantaneous overcurrent or time-overcurrent elements can be allowed trip the SEL-551c relay. However, the setting of the relay trip logic will determine how the relay responds to different overcurrent conditions.

There are three elements which determine a relay trip shown in Table 3.6. The most critical of these is the element TR which specifies the trip conditions. TR is a logical equation made up of relay word bits from instantaneous and time-overcurrent elements. If the appropriate overcurrent elements satisfy the trip equation $\mathrm{TR}$, the relay will assert the trip signal. In other words, at any time if $\mathrm{TR}=\operatorname{logical} 1$, the relay asserts the TRIP signal. The second element which impacts the relay trip is TDURD, which is the minimum trip duration time. This element specifies the minimum number of cycles for which the trip signal is asserted. Figure 3.4.1 shows TR, TDURD, and TRIP during an example relay trip operation. The first signal is $\mathrm{TR}$ and is the output of the trip control equation. The second signal is the output of the minimum trip duration timer. Note that once a rising edge of TR is detected, the output of the minimum trip duration timer maintains logical 1 for the number of cycles specified by TDURD. The third signal is the actual TRIP output. This is the logical OR-combination of the previous two signals. 
Table 3.5: Elements Which Determine Relay Trip [16

\begin{tabular}{|c|c|}
\hline Element & Function \\
\hline \hline TR & Trip Conditions (control equation) \\
\hline ULTR & Unlatch Trip Conditions \\
\hline TDURD & Minimum Trip Duration Time \\
\hline
\end{tabular}

The final element involved with a relay trip is ULTR. This element is a logical equation similar to TR, however, it specifies the unlatch trip conditions. Once the relay TRIP signal has been asserted, it remains asserted until $\mathrm{TR}=$ logical 0 , the minimum trip duration timer $=$ logical 0 , and ULTR $=$ logical 1 . Figure 3.4 .2 details the trip operation logic. Note that elements corresponding to either push button or serial inputs are not utilized in this work.

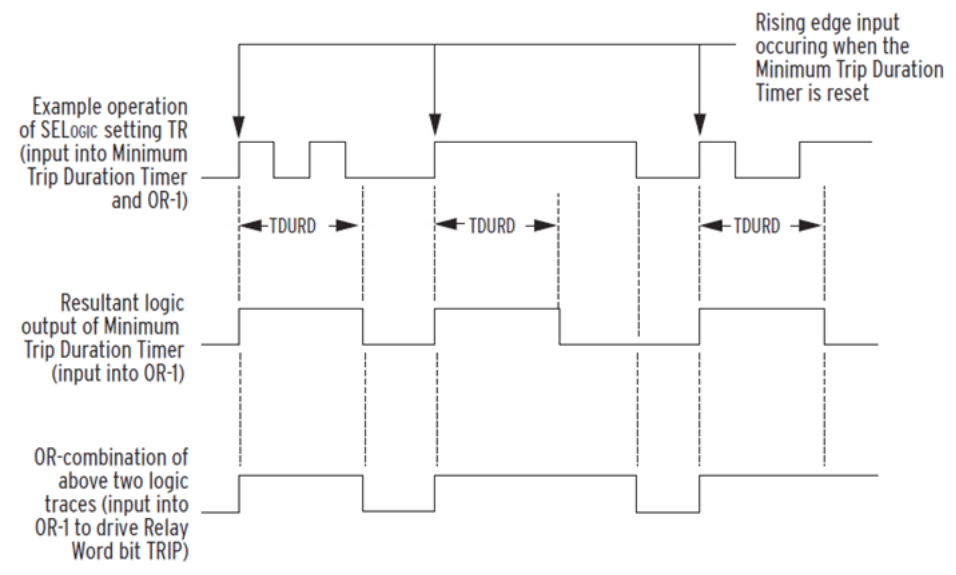

Figure 3.4.1: Example SEL-551c trip operation [16]

For example, for the factory settings of the SEL-551c, the control equation TR $=51 \mathrm{P} 1 \mathrm{~T}+51 \mathrm{G} 1 \mathrm{~T}+50 \mathrm{P} 1 * \mathrm{SH} 0+\mathrm{LB} 3$, TDURD is set to 9.0 cycles, and ULTR $=!(51 \mathrm{P} 1+51 \mathrm{G} 1)$. With these settings, the control equation will assert logical 1 from four possible elements. First, either of the time-overcurrent elements 51P1T and 51G1T can assert TR directly. The instantaneous element 50P1 can also assert TR, however, it is supervised by the relay word bit SH0. This means that the instantaneous element can only assert $\mathrm{TR}$ if $\mathrm{SH} 0=1$, i.e., the reclosing relay is at shot 0 . After the first reclose cycle, 50P1 can no longer assert TR. The element LB3 
corresponds to a manual trip from a push button on the front panel of the relay. Finally, the ULTR equation specifies that the TRIP signal will only deassert when both time-overcurrent elements deassert.

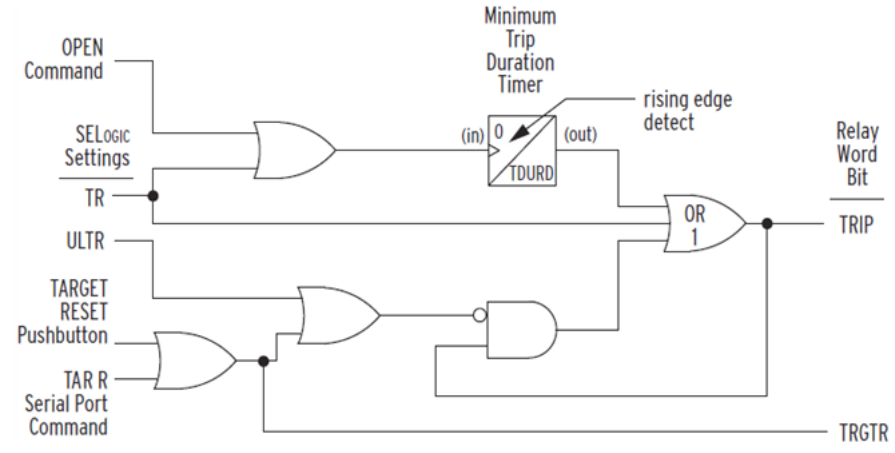

Figure 3.4.2: SEL-551c trip logic [16]

\subsection{Reclosing Logic}

The previous section described the relay trip operation logic. This section first describes the close operation logic and concludes with the explanation of the reclose function of the SEL-551c.

\subsubsection{Close Logic}

Similar to the relay trip logic, there are four elements that impact the relay close function shown in Table 3.6. If these elements meet the appropriate conditions, then the relay will output a CLOSE signal. The first element is CL which specifies the close conditions. Similar to the trip conditions TR, CL is a logical equation which will be asserted when its elements are satisfied. The second element is ULCL or the Unlatch Close Conditions. This element is a logical equation similar to ULTR which specifies under which conditions the CLOSE signal may deassert. The element 52A is the circuit breaker status. This element receives an external input which indicates whether the circuit breaker is open or closed. The last element involved is CFD or Close Failure Time. For the purposes of this work, CFD will be disabled and is not be considered in future discussions. 
Table 3.6: Elements Which Determine Relay Close

\begin{tabular}{|c|c|}
\hline Element & Function \\
\hline \hline CL & Close Conditions \\
\hline ULCL & Unlatch Close Conditions \\
\hline $52 \mathrm{~A}$ & Circuit Breaker Status \\
\hline CFD & Close Failure Time \\
\hline
\end{tabular}

The complete logic of the relay close operation is shown in Figure 3.5.1. In order for the relay to assert the CLOSE signal, all four prerequisite conditions must be true and either of two critical conditions must be true. The four prerequisite conditions are the following: Unlatch Close must not be asserted (ULCL $=$ logical 0$)$, the circuit breaker must be open $(52 \mathrm{~A}=$ logical 0$)$, a rising edge is not detected from the reclose initiation condition (detailed in the next section), and a close failure does not exist. Given that the four prerequisite conditions for the close operation are met, a CLOSE signal will be asserted if CL makes a rising edge transition or if the reclosing relay interval times out. The primary trigger to assert the relay CLOSE signal in this work will be the reclosing relay interval time-out which will be discussed in the next section. Once the CLOSE signal is asserted, it will remain asserted until either the Unlatch Close condition asserts (ULCL $=$ logical 1$)$, the circuit breaker closes $(52 \mathrm{~A}$ $=$ logical 1), reclose initiation condition makes a rising edge transition, or the close failure timer times out.

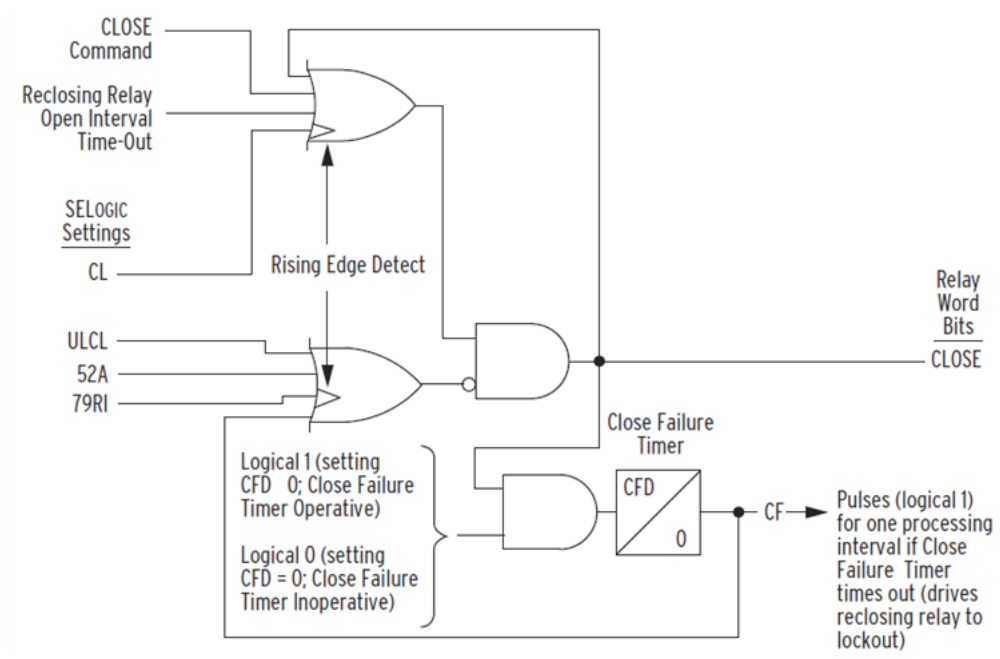

Figure 3.5.1: SEL-551c close logic [16] 
For example, using the factory settings of the SEL-551c, the close conditions are set to $\mathrm{CL}=\mathrm{LB} 4$ (manual close from relay front panel), ULCL $=$ TRIP (prevents CLOSE signal from asserting when TRIP signal is asserted), and 52A = IN1 (external input). Note that CL is set to a manual input and will not be utilized for autoreclosing in this work. Therefore, the relay reclose operation will be primarily initiated by the reclosing relay interval time-out.

\subsubsection{Autoreclosing Operation}

In previous sections, the TRIP and CLOSE operation logic of the SEL-551c have been described. The ultimate goal of this section is to use the knowledge of the operation of these functions to describe how the autoreclosing operation is accomplished. The SEL-551c implements its autoreclosing operation as shown in Figure 3.5.2. Essentially, when the SEL-551c is programmed to provide the autoreclosing function, there are three states which the relay can be in. The Lockout and Reset States are intermediate states and the Reclose Cycle State is the only state where reclosing can occur. 


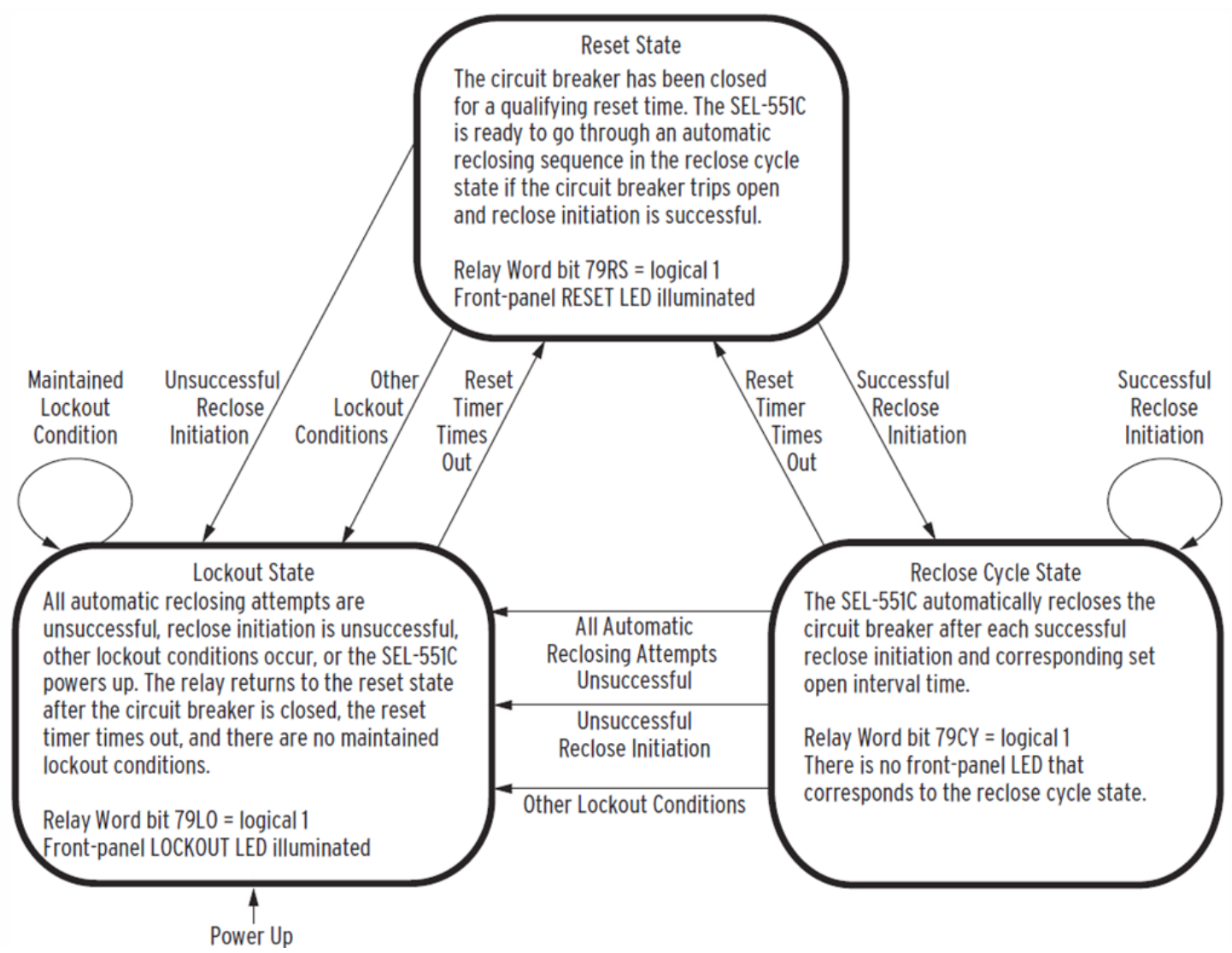

Figure 3.5.2: Automatic reclosing logic [16]

When the relay is first powered on and connected to the system, it will be in the Lockout state. In this state, the relay is locked out and waits to sense circuit breaker closure, the reset timer to time out, and that no other maintained lockout conditions are present. In other words, there is a programmable internal relay element called the Reset Timer. If the relay is in the Lockout State and the circuit breaker is closed with no other lockout conditions present the Reset Timer begins timing. If it reaches the specified time, the relay will transition to the next state, the Reset State.

The Reset State can be thought of as a standby condition. The relay is ready to perform the reclosing sequence, however, it waits for the reclose initiation, which is programmed to be the TRIP signal. When the relay senses an overcurrent condition and the TRIP signal is asserted, the relay transitions to the Reclose Cycle State. In this state, the duration of the relay reclose intervals are programmed using elements 79OI1, 79OI2, 79OI3, and 79OI4, corresponding to reclose intervals 1-4 respectively. The number of reclosing shots the relay will attempt is determined by how many of 
these elements are set to non-zero values. For example, if $79 \mathrm{OI} 1$ and $79 \mathrm{OI} 2$ are set to a non-zero value specified in number of cycles and the remaining elements set to zero, the relay will perform 3 reclosing shots separated by two reclose intervals. In this way, the number of reclosing shots and the length of the reclosing intervals can be set. If a fault is temporary and clears before all reclosing shots are attempted, the relay will return to the Reset State. If the fault is permanent and is not cleared after all reclosing shots are attempted, the relay will return to the Lockout State and lockout the circuit breaker. Note that the complete description of the reclosing operation involves more detail than described above. However, only aspects which are pertinent to this work have been described. For a complete discussion of the SEL-551c autoreclosing operation, see [16].

\subsection{SEL Software}

Three software available from SEL are utilized. The first this SEL AcSELator Quicket. This software allows the user to easily program the SEL-551c settings as well as trip and close equations. A screen shot of the interface is shown in Figure 3.6 .1 . 


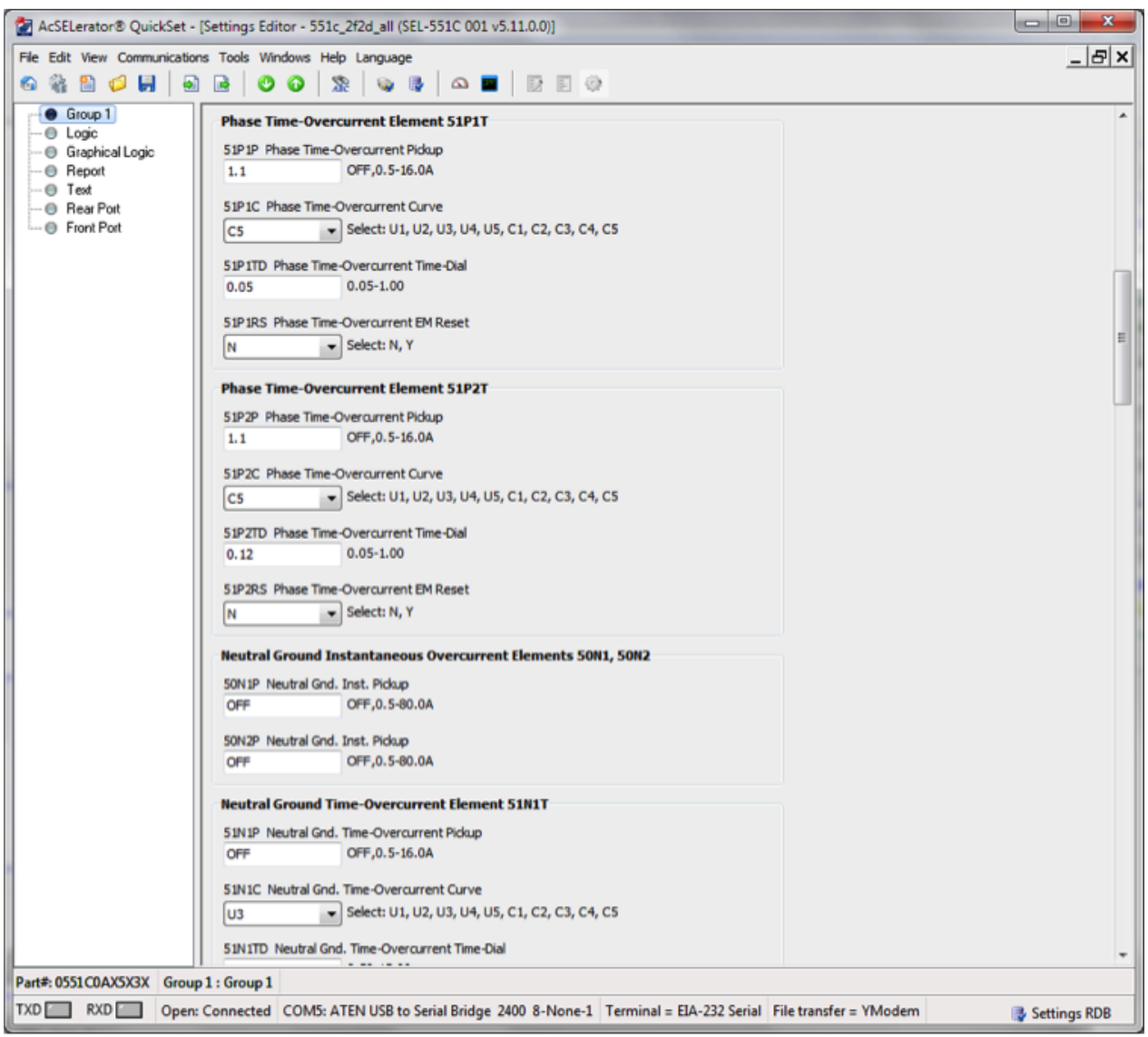

Figure 3.6.1: Specifying SEL-551c relay settings using SEL AcSELerator QuickSet

The second software utilized is SEL 5401. This enables the user to program the waveforms to be output by the SEL-AMS signal generator. The interface allows the input of current magnitudes and phase angles in addition to contact closure and state transition conditions. An example screen shot is shown in Figure 3.6.2. 


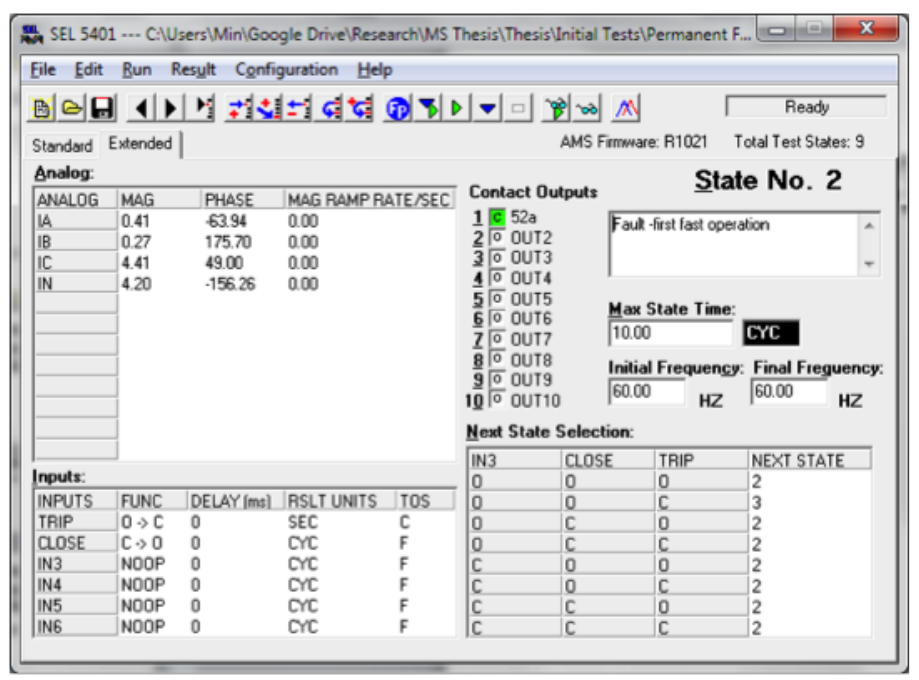

Figure 3.6.2: Simulating a single line-to-ground fault using SEL 5401

The last software utilized is the SEL event recorder capability in the the relay itself. This can capture multiple events for a maximum duration of 15 cycles each. An example captured reclose operation is shown in Figure 3.6.3. Another form of data provided by the relay's event capture is information associated with each event. This includes relay shot number, fault type, and fault current magnitudes. An example is shown in Figure 3.6.4. A detailed description of how this software is utilized will be provided in Chapter 5 . 


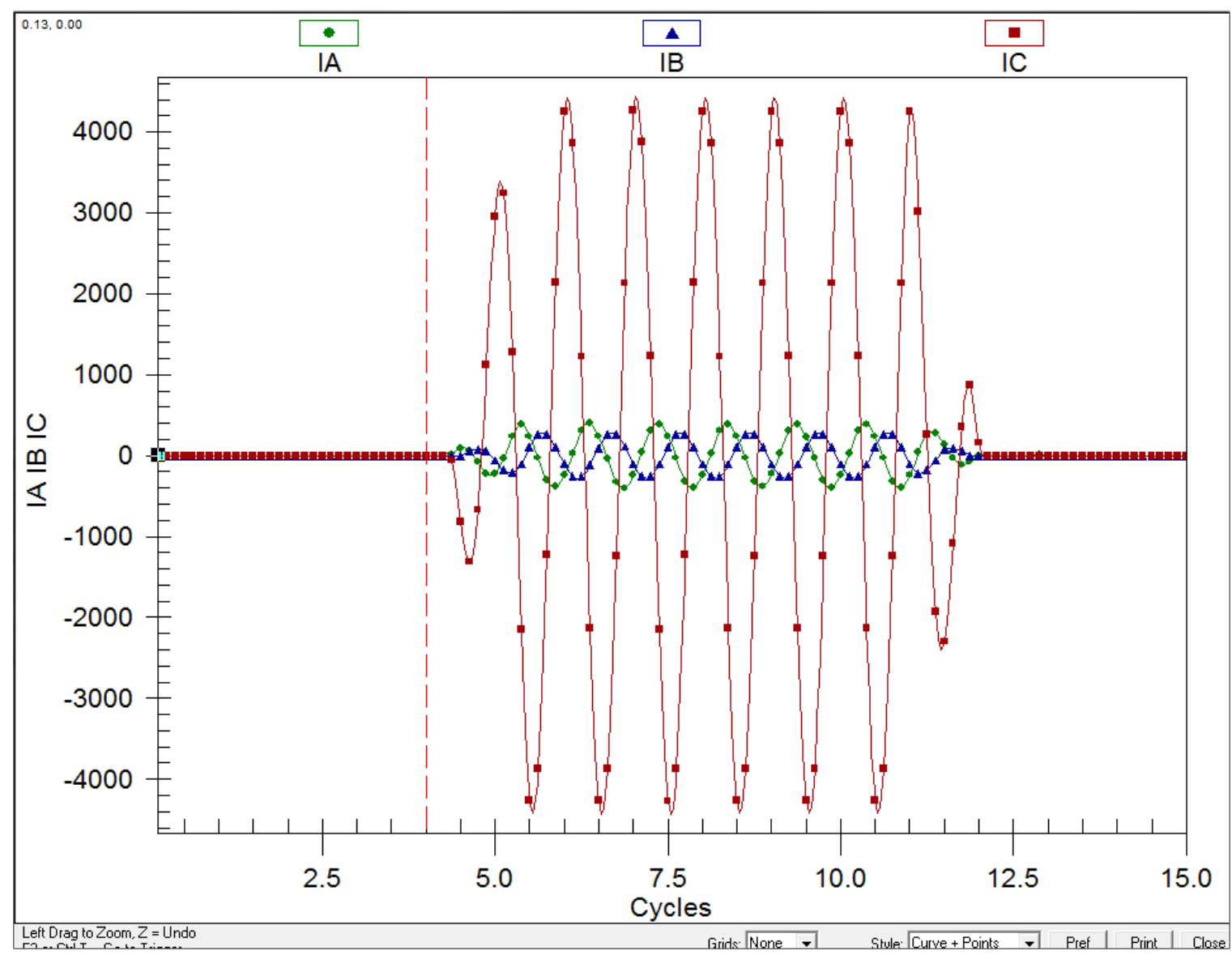

Figure 3.6.3: Event capture waveform from SEL-551c relay

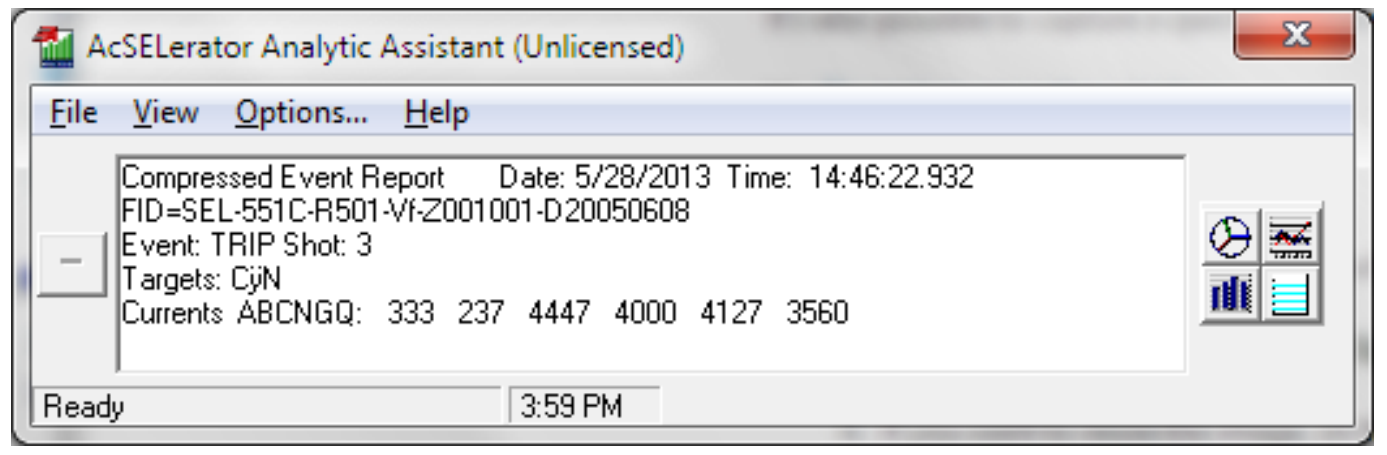

Figure 3.6.4: Event capture data from SEL-551c relay 
4

\section{Development of PSCAD/EMTDC Simulation Model}

Developing a simulation model of a recloser or any device is important to determine how the component and associated system will behave under various operating conditions. Distribution circuits are often modeled in PSCAD/EMTDC software. Although the software includes models of basic protective devices and functions, a built-in recloser model is not available. This chapter will discuss the development of a PSCAD simulation model for the reclosing function of the SEL-551c relay. The purpose of the model is to emulate the reclosing logic and operating performance of the relay in PSCAD. The first component is the signal processing block which will take time-domain current signals from the distribution circuit model and pass them to the TCC curve blocks. The next part of the model are the TCC curve blocks. These components implement the fast and slow inverse time-overcurrent protective functions of the recloser. The next task will be to develop a logic model from existing logic components to emulate the SEL-551c reclosing logic. The last component is the circuit breaker control. This part of the model will use the TRIP and CLOSE signals from the recloser logic and appropriately control the circuit breaker. Together, the components will be combined to complete the model of the SEL-551c reclosing function. The block will be tested and validated in a distribution circuit model based on a real world circuit. In [14], simulations of the reclosing operation during a fault were done by manually specifying the relay operating times based on the TCC curve equation. This work proposes to develop a relay model in PSCAD which simulates the actual operation of the SEL-551c relay. Relay TCC curve equations and other settings will be input to the PSCAD component. 


\subsection{Distribution Circuit Model}

The test distribution circuit one-line diagram is shown in Figure 4.1.1 and the PSCAD model is shown in Figure 4.1.2. Note that this is a relatively small and simple distribution circuit, allowing one to limit the number of variables. The equivalent source is specified at $12 \mathrm{MVA}$ and operates at $115 \mathrm{kV}$. The radial topology and lack of distributed generation allows for a straightforward analysis. Temporary and permanent single line-to-ground faults, at locations $\mathrm{A}$ or $\mathrm{B}$, will be studied as this type of fault is most commonly encountered on a distribution system.

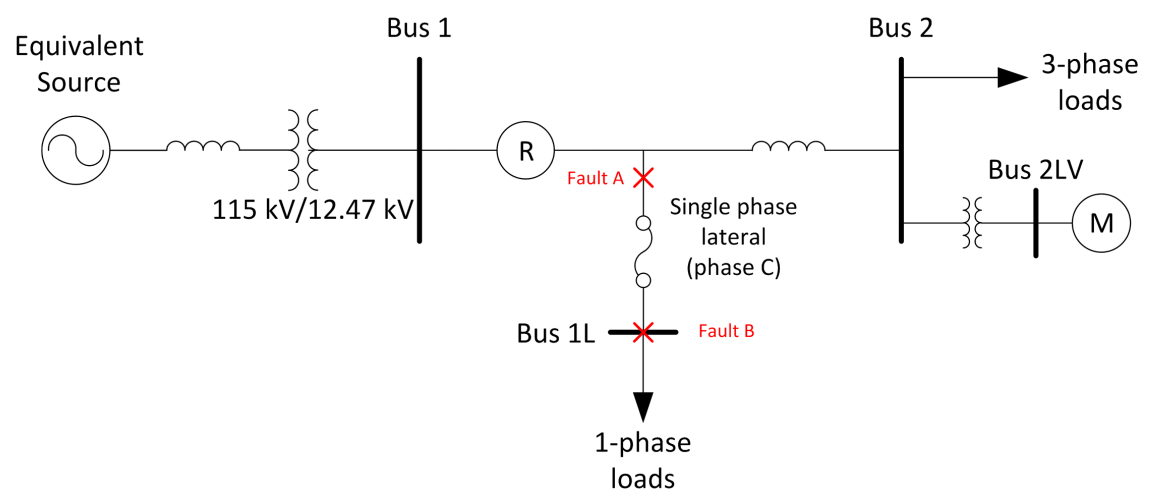

Figure 4.1.1: One-line diagram of distribution circuit model

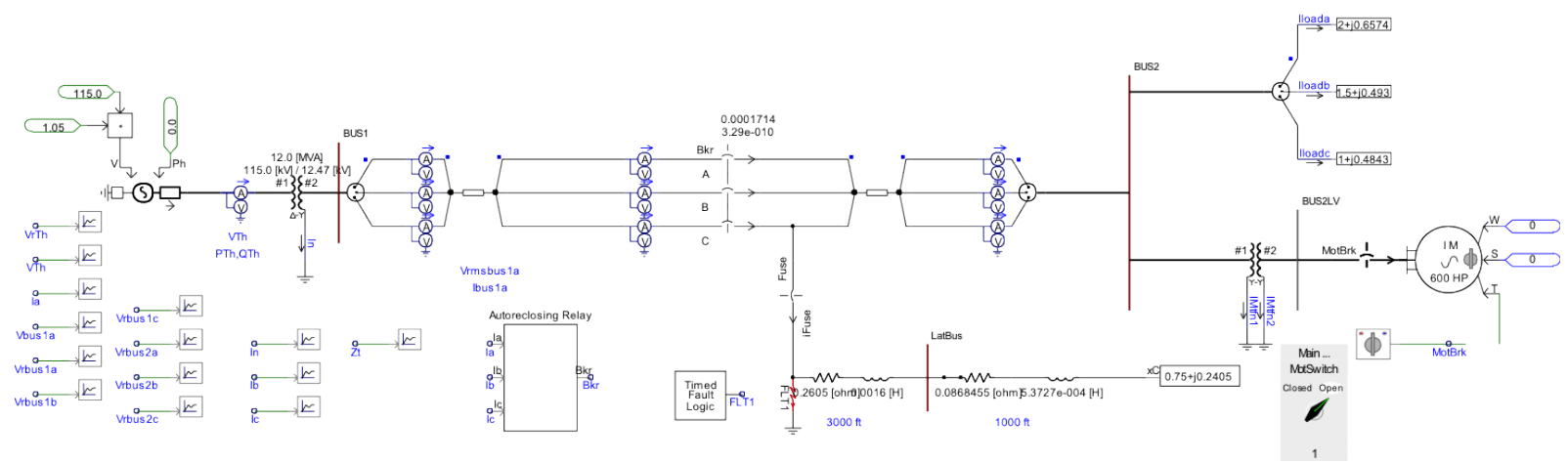

Figure 4.1.2: PSCAD distribution circuit model 


\subsection{Relay Model Development}

The goal of this model is to develop a PSCAD component that will control the built-in circuit breaker model to emulate the reclosing function of the SEL-551c. The model is shown in Figure 4.2.1 and will be described from left to right in the following sections. The objective is to operate the reclosing relay in a fuse saving scheme, i.e., operating on the fast curve for the first two reclose operations and on the delayed curve for the last two operations.

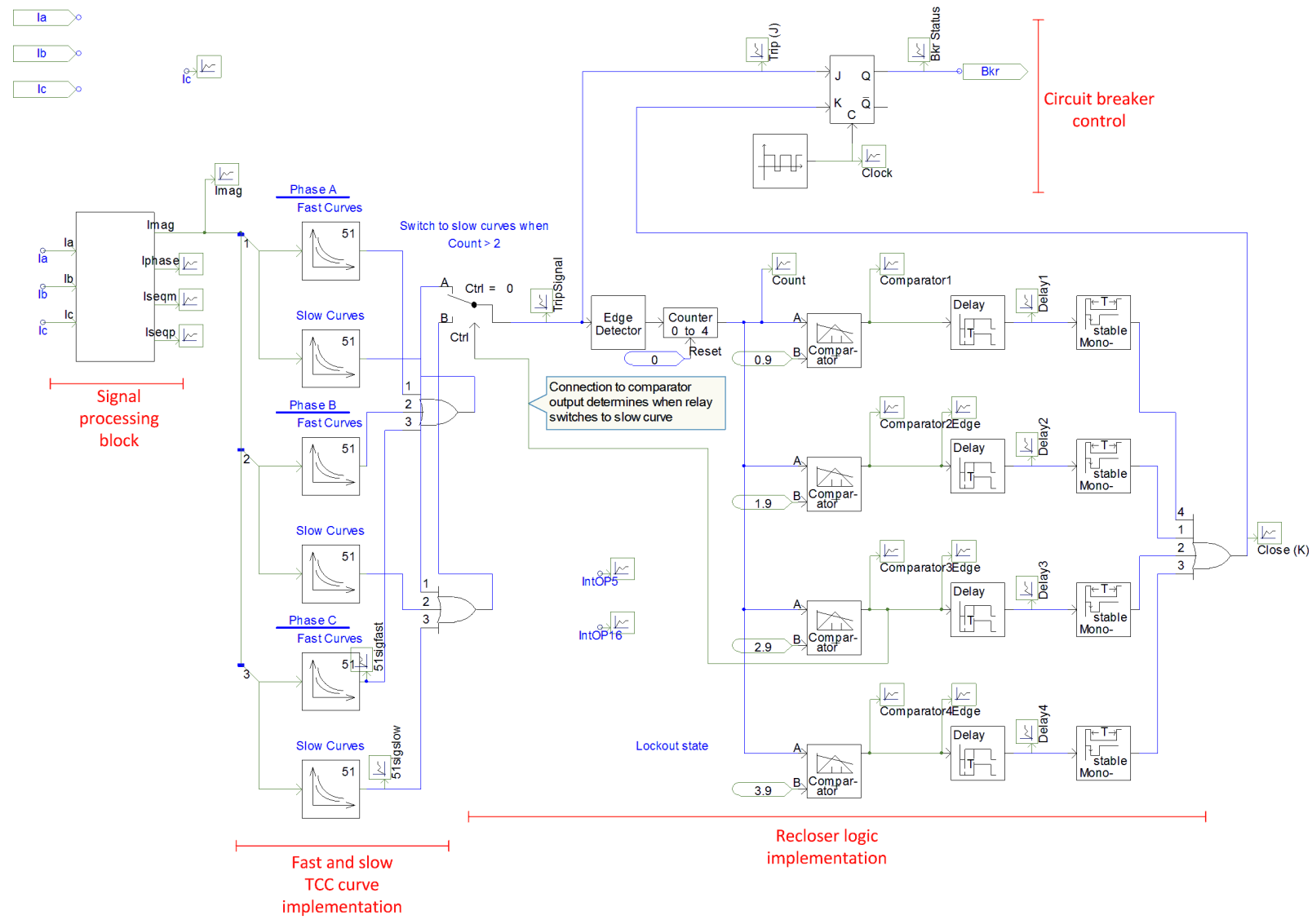

Figure 4.2.1: PSCAD reclosing relay model 


\subsubsection{Signal processing block}

The first component developed is the signal processing block which will take timedomain current signals from the distribution circuit model and pass them to the TCC curve blocks. This block is shown on the left side of Figure 4.2.1 with inputs from $I_{a}, I_{b}$, and $I_{c}$. Using the FFT block available in PSCAD, the phasor magnitude of current for the fundamental component of each phase is passed on. Additionally, although not utilized by the TCC curve blocks, the fundamental component phase angle and sequence component magnitude and phase angle are output and can be observed.

\subsubsection{TCC Curve Implementation}

The next task in developing the relay model is to implement the inverse timeovercurrent protective function of the SEL-551c. Inverse time-overcurrent function blocks are built-in to PSCAD. The blocks allow for the user to choose the settings for standard TCC curve types. Additionally, more customized curves can be implemented in these blocks by manually choosing the variables in the generic TCC equation below.

$$
\mathrm{t}=\mathrm{TD} \cdot\left(\frac{\mathrm{A}}{\mathrm{M}^{\mathrm{p}}-1}+\mathrm{B}\right)+\mathrm{K}
$$

If the input to the block exceeds the specified pickup current, then the block imposes a time delay based on the specified TCC curve before asserting a high signal at the output. In this manner, the curves available for the SEL-551c shown in Table 3.3 and 3.4 can be implemented in the relay model. For each phase, two curves (fast and slow) are implemented using these built-in inverse time-overcurrent blocks with different TCC equations. The output from the fast curves of all three phases are input to an OR gate. Similarly, the output from the three slow curves are also input to a separate OR gate. If there is a fault on any phase, the output of either OR gate will go high based on whether the fast or slow curve is specified. 


\subsubsection{Recloser Logic Implementation}

The next part of the model implements the recloser logic of the relay. The OR gate outputs are passed to a two-selector control switch. The fast curve operation is connected to selection $\mathrm{A}$ and the slow curve operation is connected to selection B. The switch is set to selection A until a control signal is received. The logic circuit to the right of the selector switch counts the number of shots and changes the selector switch from fast curve operation to slow curve operation after two shots are counted. Additionally, the output of the selector switch also acts as the TRIP signal to the circuit breaker.

In order to count the number of reclosing shots, the output from the selector switch is passed to an edge detector connected to the built-in counter block. Whenever either the fast or slow curve output times out, the counter will be incremented to an integer value between 0 and 4 . However, because this counter value will change after each increment, the output is passed to four comparators. The output of each comparator will go high and remain high after the shot counter has observed each reclosing shot. In other words, the output of the first comparator will go high after shot count 1, the output of the second comparator will go high after shot count 2, and similarly for all four. The output from the third comparator is used to activate the control switch; after two reclosing shots are counted, the model will operate on the slow curves.

The next step is to implement the CLOSE signal. In the SEL-551c logic, after a trip operation, the relay waits for the specified reclose interval time before attempting to reclose. This reclose interval can be thought of as a delayed CLOSE signal. Therefore, the output of the comparator will be delayed using the built-in delay and then monostable blocks. After each shot counter increment, the corresponding comparator will go high. The delay block implements the reclose interval delay and the monostable block changes a constant signal into a pulse (explained later for the circuit breaker input). The pulse output from each monostable block will act as one CLOSE signal. Each pair of these blocks implements a corresponding reclose interval. That is, the first pair of delay and monostable blocks implements the first reclose interval, the second pair implements the second reclose interval, and the third pair implements the third reclose interval. Note that because the relay is operating in a two-fast, two-delayed operation, the relay will lockout after the fourth shot. The 
outputs from each of these delay-monostable block pairs is input to an OR gate. Each pulse from the monostable blocks pass through the OR gate as the CLOSE signal.

\subsubsection{Circuit Breaker Control}

At this point, the TRIP and CLOSE signals have been implemented in PSCAD. The TRIP signal is output from the selector switch and the CLOSE signal is output from the monostable block as pulses. However, these signals cannot be simply passed to the circuit breaker model in PSCAD to implement the reclosing function. The standard circuit breaker model is controlled by one external binary signal. Initially the circuit breaker is closed by default. An input signal of 0 will maintain the breaker closed and an input of 1 will trip the breaker open. In order to achieve the desired reclosing operation using the developed TRIP and CLOSE logic, the states in Table 4.1 must be implemented. Note that a breaker status of 0 corresponds to the closed state and 1 corresponds to the open or tripped state. Additionally, the state where both TRIP and CLOSE are simultaneously asserted is not allowed.

Table 4.1: Desired PSCAD Circuit Breaker Operation

\begin{tabular}{|c|c|c|}
\hline TRIP & CLOSE & Breaker Status \\
\hline \hline 0 & 0 & 0 \\
\hline 0 & 1 & 0 \\
\hline 1 & 0 & 1 \\
\hline 1 & 1 & not allowed \\
\hline
\end{tabular}

Note: breaker status of 0 corresponds to the closed state and 1 corresponds to the open or tripped state.

Using the J-K flip-flop block in PSCAD provides a simple way of implementing the reclosing operation. The truth table in Table 4.2 shows how TRIP and CLOSE are used as inputs to $\mathrm{J}$ and $\mathrm{K}$ respectively to achieve the desired logic. Under normal operating conditions, the circuit breaker is closed and both TRIP and CLOSE signals are 0 . In this state, the flip-flop will maintain in the closed state. When a fault is sensed and the TRIP signal is asserted, the breaker is opened. During the subsequent reclose interval, both TRIP and CLOSE are low which maintains the opened state. After the specified reclose interval, the CLOSE signal is asserted and the breaker is 
reset to the closed state. As before, the state where both J and K are simultaneously asserted is not allowed.

Table 4.2: J-K Flip-Flop Truth Table Implementation

\begin{tabular}{|c|c|c|c|c|}
\hline J (TRIP) & K (CLOSE) & Q (Breaker Status) & $\overline{\mathrm{Q}}$ & Description \\
\hline \hline 0 & 0 & $\mathrm{Q}$ & $\overline{\mathrm{Q}}$ & hold \\
\hline 0 & 1 & 0 & 1 & reset \\
\hline 1 & 0 & 1 & 0 & set \\
\hline 1 & 1 & $\overline{\mathrm{Q}}$ & $\mathrm{Q}$ & toggle (not allowed) \\
\hline
\end{tabular}

Note: breaker status of 0 corresponds to the closed state and 1 corresponds to the open or tripped state.

\subsection{Simulation Results}

To validate the accuracy of the recloser model, four fault scenarios were considered shown in Table 4.3. Each fault scenario results in a different recloser sequence. In each case, a single line-to-ground fault was placed at either location A or location $\mathrm{B}$ on the the single phase lateral in Figure 4.1.1 on phase $\mathrm{C}$ at $1.00 \mathrm{sec}$ into the simulation. The recloser model is set to operate in fuse saving mode, with two fast and two delayed operations. The I.E.C. short-time inverse (C5) curve, Equation 4.3.1, is utilized for both fast and delayed curves in the model. The time-delay setting is changed to achieve the two operating times, where TD is the time-delay setting and $\mathrm{M}$ is multiples of pickup.

$$
\mathrm{t}_{\mathrm{p}}=\mathrm{TD} \cdot\left(\frac{0.05}{\mathrm{M}^{0.04}-1}\right)
$$

Table 4.3: Summary of Fault Scenarios Tested

\begin{tabular}{|c|c|c|c|}
\hline Fault Scenario & Type & Location & Reclosing Sequence \\
\hline \hline 1 & temporary & B & one fast operation \\
\hline 2 & temporary & B & two fast operations \\
\hline 3 & permanent & B & two fast operations, fuse clears fault \\
\hline 4 & permanent & A & two fast and two delayed, recloser lockout \\
\hline
\end{tabular}


In order to observe different reclosing sequences, the type of fault, duration, and location is changed. First, two temporary faults, downstream of the fuse, with varying durations are tested (Scenarios 1 and 2). This results in one fast operation and two fast operations (depending on the duration of the fault) before the fault self-clears. In both of these cases, the recloser operates before the fuse clears. Next, two permanent faults are tested (Scenarios 3 and 4 ). The first is a permanent single line-to-ground fault on the single phase lateral, downstream of the fuse. This scenario results in two fast operations and the fuse clearing the fault. Note that after the fuse clears the fault, the current on phase $\mathrm{C}$ will be lower than the pre-fault phase $\mathrm{C}$ current. The last scenario is a permanent fault on phase C, upstream of the fuse. This scenario results in two fast operations and two delayed operations followed by recloser lockout. The resulting simulation waveforms of the four reclosing sequences are shown in Figure 4.3.1. A detailed analysis and comparison with the SEL-551c relay will be performed in Chapters 5 and 6 . It will be shown in these following chapters that, by comparing relay operating times and reclose intervals to the TCC curves, the accuracy of the recloser model is acceptable.
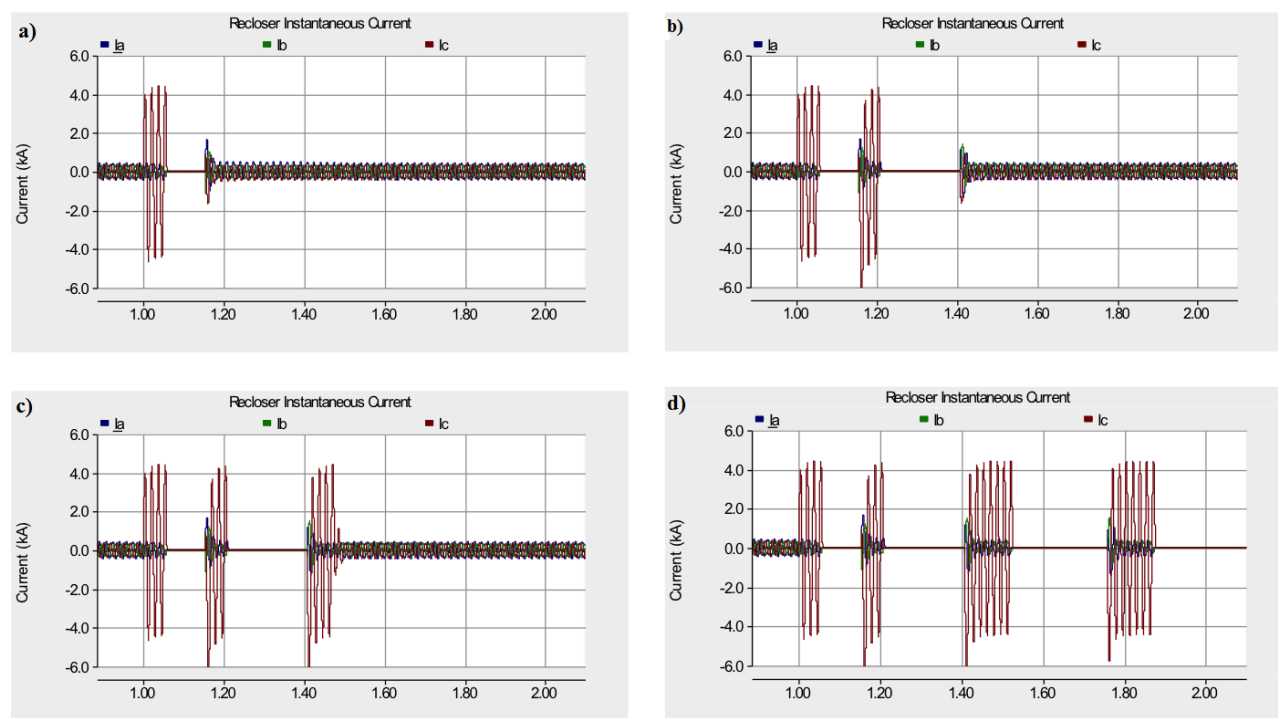

Figure 4.3.1: Simulation results and corresponding reclosing sequences: a) one fast operation, b) two fast operations, c) two fast operations, fuse clears fault, d) two fast and two delayed operations, recloser lockout 


\section{5 \\ Reproducing Simulation Results in Hardware}

In Chapter 3, an overview of the SEL-551c hardware and corresponding software was provided. In Chapter 4, a software simulation model in PSCAD was developed to model the recloser behavior. Although the model provides a good approximation of how a reclosing relay may operate based on TCC settings, the ultimate goal is to compare the performance of the simulation model with the performance of a real world relay. This chapter will describe how an experiment is conducted using SEL551c test interface to simulate the same fault conditions on the SEL-551c as simulated in the PSCAD model. Results from the experiment and PSCAD simulations are presented. The goal of the experiment is to replicate the fault conditions simulated in PSCAD in Chapter 4. As before, four fault conditions resulting in four different reclosing sequences will be tested. The PSCAD distribution system model and fault simulations provide a good approximation of how this real world system would behave under various faults. This experiment will attempt to approximate how the SEL-551c would actually operate during those same fault conditions on a real world distribution system.

The methodology of this experiment is to first simulate four single line-to ground fault scenarios in the PSCAD model using the recloser model developed in Chapter 4. The relay in the model will be set to operate in fuse saving mode with two fast and two delayed operations. Each scenario will result in a distinct reclosing sequence. The recloser settings will be the same for each scenario. The different reclosing sequences will be achieved by changing the type, duration, and location of the faults. In order to test how the SEL-551c performs under the same conditions, the SEL-551c will 
be programmed with the same settings (TCC curve type, time-delay setting, pickup current, etc.). Using the SEL test interface, the fault current waveform from the PSCAD model for each scenario will be replicated and input to the SEL-551c relay. Relay performance will then be evaluated by measuring when the TRIP and CLOSE signals are asserted by the relay and also by analyzing the event capture waveforms from the relay.

\subsection{SEL Test Interface}

Schweitzer relays can be tested using the SEL-AMS (Adaptive Multichannel Source) which is essentially a signal generator. This signal generator can be programmed to generate low-level sinusoidal signals which match a desired fault current waveform. The SEL-AMS can also sense inputs to monitor relay contacts and itself has output contacts to drive relay logic. LEDs on the front panel indicate sensed input status or contact output status. This allows one to easily monitor the status of the relay contacts and output contacts during the experiment. Using the SEL 5401 test software, one can simulate a reclosing event by programming different states for the SEL-AMS signal generator to output. In this experiment, the autoreclosing and event capture capabilities of the SEL-551c were tested using four autoreclosing sequences.

\subsubsection{Setting the Relay}

Before any experiment can be performed, the SEL-551c relay must be programmed with the desired settings. This task can easily be performed using the SEL AcSELerator Quickset software. By connecting the relay to any PC that has the software installed, one can set the desired settings detailed in Chapter 3. For example, the factory settings for this relay are shown in Table 5.1. Note that the trip signal logic equation in these factory settings will not operate as a recloser with a fast and delayed curve. 
Table 5.1: Summary of SEL-551c Factory Settings

\begin{tabular}{|c|c|c|}
\hline Element Name & Setting Value & Description \\
\hline CTR & 120 & Phase $\left(\mathrm{I}_{\mathrm{A}}, \mathrm{I}_{\mathrm{B}}, \mathrm{I}_{\mathrm{C}}\right)$ CT Ratio \\
\hline CTRN & 120 & Neutral $\left(\mathrm{I}_{\mathrm{N}}\right)$ CT Ratio \\
\hline TDURD & 9.0000 сус & Minimum Trip Duration Time \\
\hline TR & $51 \mathrm{P} 1 \mathrm{~T}+51 \mathrm{G} 1 \mathrm{~T}+50 \mathrm{P} 1 * \mathrm{SH} 0+\mathrm{LB} 3$ & Trip Signal Logic \\
\hline CL & LB4 & Close Conditions \\
\hline $50 \mathrm{P} 1 \mathrm{P}-50 \mathrm{P} 6 \mathrm{P}$ & OFF A & Phase Inst. Pickup \\
\hline $50 \mathrm{ABCP}$ & OFF A & Single Phase Inst. Pickup \\
\hline $51 \mathrm{P} 1 \mathrm{P}$ & $16.0 \mathrm{~A}$ & Phase Time-Overcurrent Pickup \\
\hline $51 \mathrm{P} 1 \mathrm{C}$ & C5 & Phase Time-Overcurrent Curve \\
\hline 51P1TD & 0.05 & Phase Time-Overcurrent Time-Dial \\
\hline 51P1RS & $\mathrm{N}$ & Phase Time-Overcurrent EM Reset \\
\hline $51 \mathrm{P} 2 \mathrm{P}$ & $1.1 \mathrm{~A}$ & Phase Time-Overcurrent Pickup \\
\hline $51 \mathrm{P} 2 \mathrm{C}$ & $\mathrm{C} 5$ & Phase Time-Overcurrent Curve \\
\hline $51 \mathrm{P} 2 \mathrm{TD}$ & 0.12 & Phase Time-Overcurrent Time-Dial \\
\hline 51P2RS & $\mathrm{N}$ & Phase Time-Overcurrent EM Reset \\
\hline $79 \mathrm{OI} 1$ & $15.000 \mathrm{cyc}$ & Open Interval 1 Time \\
\hline $79 \mathrm{OI} 2$ & $45.000 \mathrm{cyc}$ & Open Interval 2 Time \\
\hline $79 \mathrm{OI} 3$ & $15.000 \mathrm{cyc}$ & Open Interval 3 Time \\
\hline 79RSD & 1800.000 cyc & Reset Time From Reclose Cycle \\
\hline 79RSLD & 300.000 cyc & Reset Time From Lockout \\
\hline NFREQ & $60 \mathrm{~Hz}$ & Nominal Frequency \\
\hline PHROT & $\mathrm{ABC}$ & Phase Rotation \\
\hline
\end{tabular}

Note: settings not listed in table are set to the default values or either OFF or 0 .

For the purposes of this experiment, the factory settings were modified to those shown in Table 5.2. First, the current transformer ratio was changed to 1000 in order to accommodate the fault current values obtained in the PSCAD simulations. Next, the trip signal logic equation (element TR) is modified to operate as a recloser with fast and delayed curves (fuse saving mode). The recloser will only operate on the fast curve (51P1T) for the first two shots (SH0 and SH1) and on the delayed curve (51P2T) for the third and fourth shots (SH2 and SH3). All other instantaneous overcurrent or time-overcurrent elements are set to be off.

Note that the event capture feature in the SEL-551c relay is limited to providing waveform data for only 15 cycles per TRIP or CLOSE event. Therefore, the reason behind choosing these particular TCC curve, time-delay, pickup and reclose interval 
settings are so that the fault current persists just long enough for the 15 cycle event capture to collect data for the entire fast or delayed operation. Particularly if the time-delay settings are too high, the fault current will persist for longer than 15 cycles and it will not be possible to determine the duration of the fault from the waveform data alone. It will be assumed that the fuse on the single phase lateral coordinates with the chosen fast and delayed curves. Ideally the recloser will be set to coordinate with the fuse type down stream of the recloser. However, for the purposes of this experiment, these settings are chosen so waveform data can be analyzed. Note that the same settings are used for parts of this experiment.

Table 5.2: Summary of SEL-551c Settings For All Experiments

\begin{tabular}{|c|c|}
\hline Element Name & New Setting \\
\hline \hline CTR & 1000 \\
\hline TR & $51 \mathrm{P}^{*}{ }^{*}(\mathrm{SH} 0+\mathrm{SH} 1)+51 \mathrm{P} 2 \mathrm{~T}{ }^{*}(\mathrm{SH} 2+\mathrm{SH} 3)$ \\
\hline $\mathrm{CL}$ & $\mathrm{LB} 4$ \\
\hline $50 \mathrm{P} 1 \mathrm{P}$ & $\mathrm{OFF}$ \\
\hline $51 \mathrm{P} 1 \mathrm{P}$ & $1.1 \mathrm{~A}$ \\
\hline $51 \mathrm{P} 1 \mathrm{C}$ & $\mathrm{C} 5$ \\
\hline $51 \mathrm{P} 1 \mathrm{TD}$ & 0.05 \\
\hline $52 \mathrm{P} 2 \mathrm{P}$ & $1.1 \mathrm{~A}$ \\
\hline $51 \mathrm{P} 2 \mathrm{C}$ & $\mathrm{C} 5$ \\
\hline $51 \mathrm{P} 2 \mathrm{TD}$ & 0.12 \\
\hline $79 \mathrm{OI} 1$ & 15.000 cyc \\
\hline $79 \mathrm{OI} 2$ & 45.000 cyc \\
\hline $79 \mathrm{OI} 3$ & 15.000 cyc \\
\hline $79 \mathrm{RSD}$ & 1800.000 cyc \\
\hline $79 \mathrm{RSLD}$ & 300.000 cyc \\
\hline
\end{tabular}

Note: settings not listed in table are set to the default values or either OFF or 0.

\subsubsection{Creating Test Sequence in SEL 5401}

SEL 5401 software allows the user to define up to 255 different states which the SELAMS can output. Because the SEL-551c overcurrent/reclosing relay only monitors current, the magnitude and phase angle values can be specified for three line and 
one neutral current signals in each state. Additionally, one can specify the state of the output contacts, which input signals to monitor, and frequency ramping between states. The transition from one state to another is determined by the conditions specified by the user. State transitions can be as simple as a preset time. In this simple case, the SEL-AMS will output signals associated with the state for a preset time before changing its output to the next state's specified output signal. However, because we want to observe and evaluate the relay's reclosing operation, state transitions in this experiment are also determined by relay contact outputs.

Figure 5.2.1 provides an example of how to test the SEL-551c reclosing operation for a temporary single line-to-ground fault that self-clears during the first reclose interval. As shown in the figure, the system starts in the pre-fault condition with nominal line and neutral currents. A single line-to-ground fault then occurs, which persists until the relay, operating on the fast curve, opens the breaker. The breaker stays open for the duration of the first reclose interval. When the relay recloses at the end of the interval, the temporary fault has cleared and the system returns to its normal operating condition.

To program this scenario in the SEL-AMS, a total of four states are required. State 1 is the pre-fault state. The SEL-AMS is programmed to output the pre-fault current for specified time. It will then automatically transition to State 2, the fault state. In the fault state, the SEL-AMS will output the specified fault current to the SEL-551c relay. The signal generator will stay in the fault state until it senses a relay trip contact closure. During this time, because the fault current exceeds the pickup setting of the relay, the relay's time-overcurrent element will start timing on the fast curve. When the time-overcurrent element times out, the relay will assert the TRIP signal and close the associated output contact. This contact closure will be sensed by the SEL-AMS and trigger a transition to State 3 (first reclose or open interval). The SEL-AMS will continue in this state until it senses the contact closure associated with the CLOSE signal from the relay. At this point, the signal generator will transition to State 4. By conditioning state transitions on relay contact closures, one can observe how the relay will operate for various conditions on the system and measure the performance of the relay by analyzing the time between state transitions. 


\subsubsection{Physical connections}

In order to implement this test procedure, the physical connections shown in Figure 5.1.1 must be made between the SEL-551c relay and the SEL-AMS signal generator. The relay only has two output signals, TRIP and CLOSE. When either of these signals are asserted through the relay logic, the corresponding contact will close and provide a $14.3 \mathrm{Vdc}$ signal to the corresponding input of the SEL-AMS. The SEL-AMS, on the other hand, has only one contact closure output signal and four analog output signals. Output 1 of the SEL-AMS provides the breaker status signal to the relay. This signal is used in the relay CLOSE logic as discussed in Chapter 3. The four analog output signals from the SEL-AMS provide the sinusoidal current waveforms that the relay will monitor. Note that these analog signals from the SEL-AMS are directly connected to the low-level inputs of the SEL-551c, bypassing the relay input transformers. This means that the signals generated by the SEL-AMS are on the order of milliamps as opposed the $0-5 \mathrm{amp}$ signal which would normally be output from a current transformer.

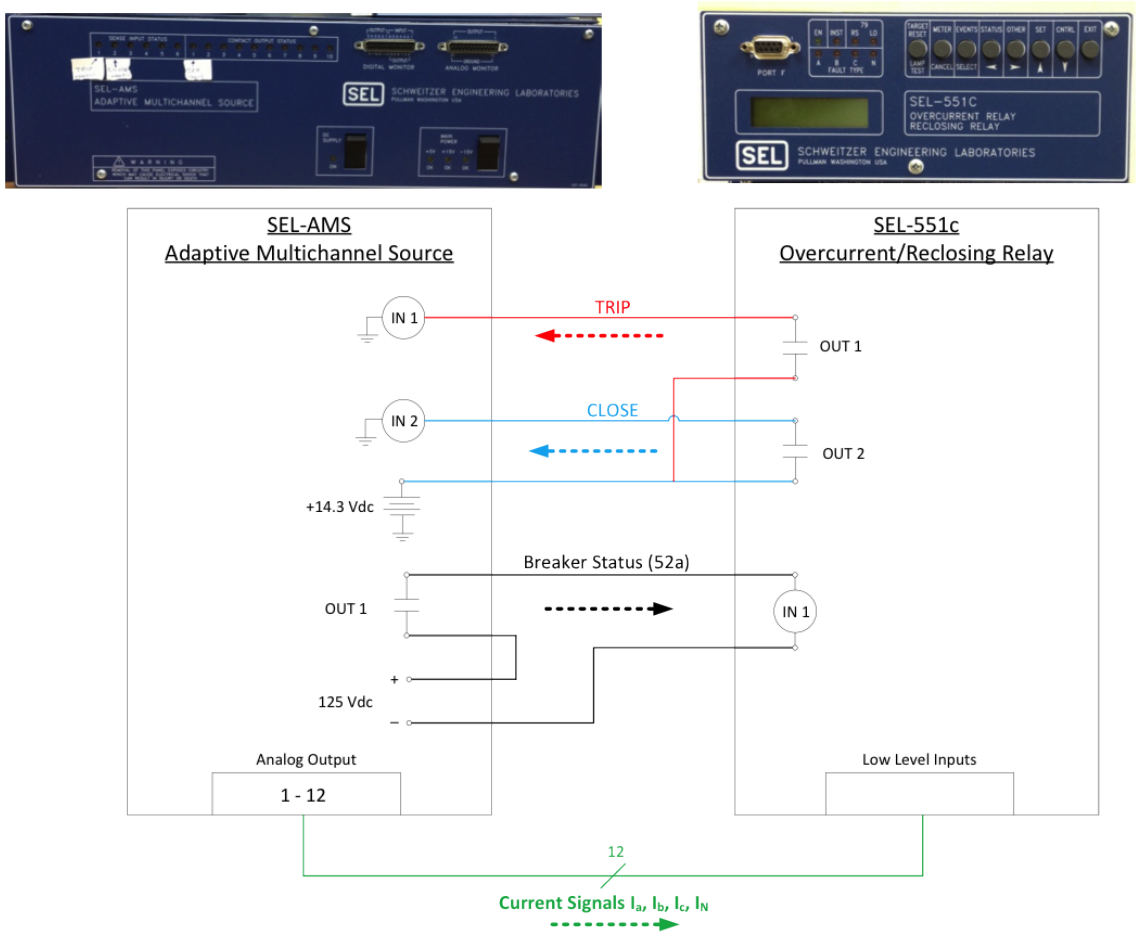

Figure 5.1.1: Physical connections between SEL-551c and SEL AMS 


\subsection{Temporary Fault: One Fast Operation}

In this scenario, a temporary single line-to-ground fault is simulated on phase $\mathrm{C}$ on the lateral, downstream of the fuse (see Figure 4.1.1). The recloser is set for two fast, two delayed operations, however the fault self-clears after the first fast operation during the first reclose interval. For both the PSCAD and SEL-551c simulations, the expected recloser sequence for one fast operation is shown in Figure 5.2.1. Note that four states are required to implement this fault condition using the SEL test interface.

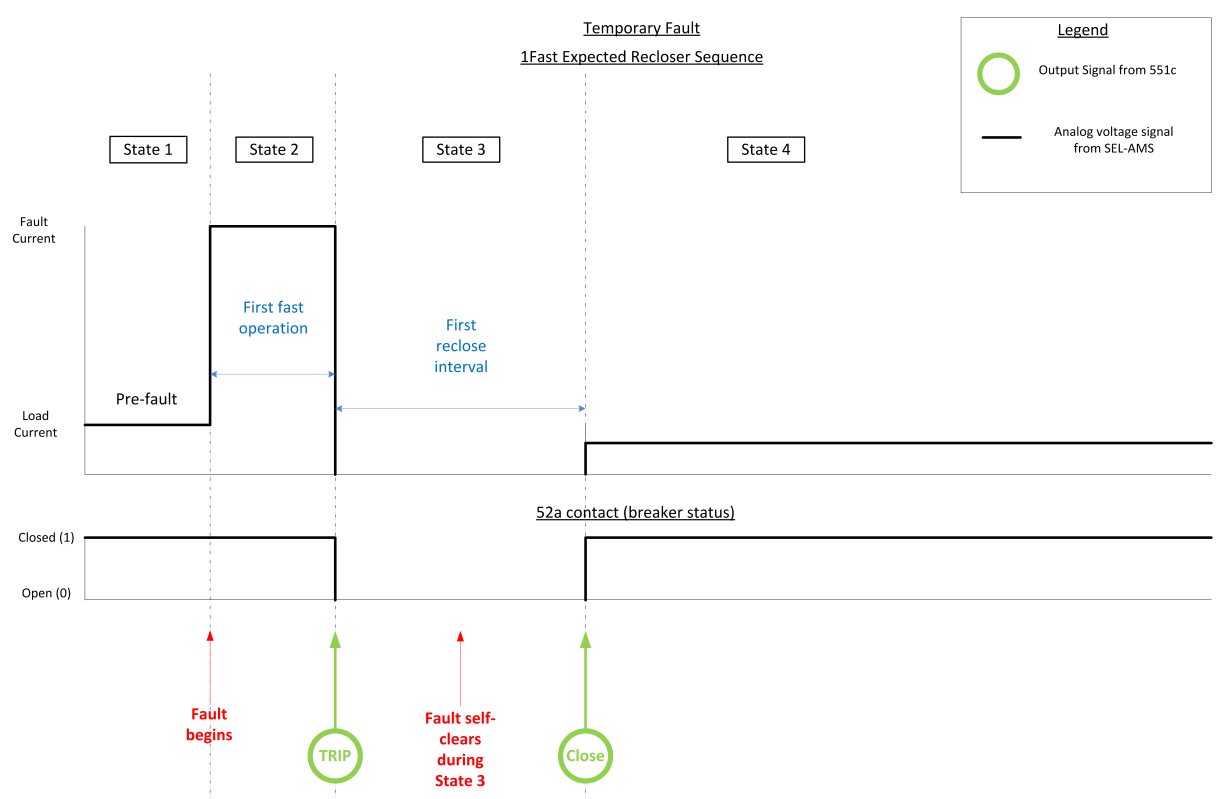

Figure 5.2.1: Expected recloser sequence for one fast operation

Results from the PSCAD simulation are provided below in Figure 5.2.2. We observe that the recloser model operates as expected. Results from the SEL-551c are shown in Figure 5.2.3. Because the event recorder is only able to capture TRIP and CLOSE events in 15 cycle windows, the data from each event has been combined and 
plotted in Matlab. Due to this limitation, we are only able to observe the duration of the first fast operation in Event 1. We are unable to observe the first reclose interval from the waveform data because it spans two different events. However, we can obtain this data from the SEL-AMS. Because the SEL test interface records when the TRIP and CLOSE signals are asserted by the relay, we can determine the length of the reclose intervals. This analysis of the operating time and reclose intervals for both SEL and PSCAD simulations will be done in Chapter 6 .

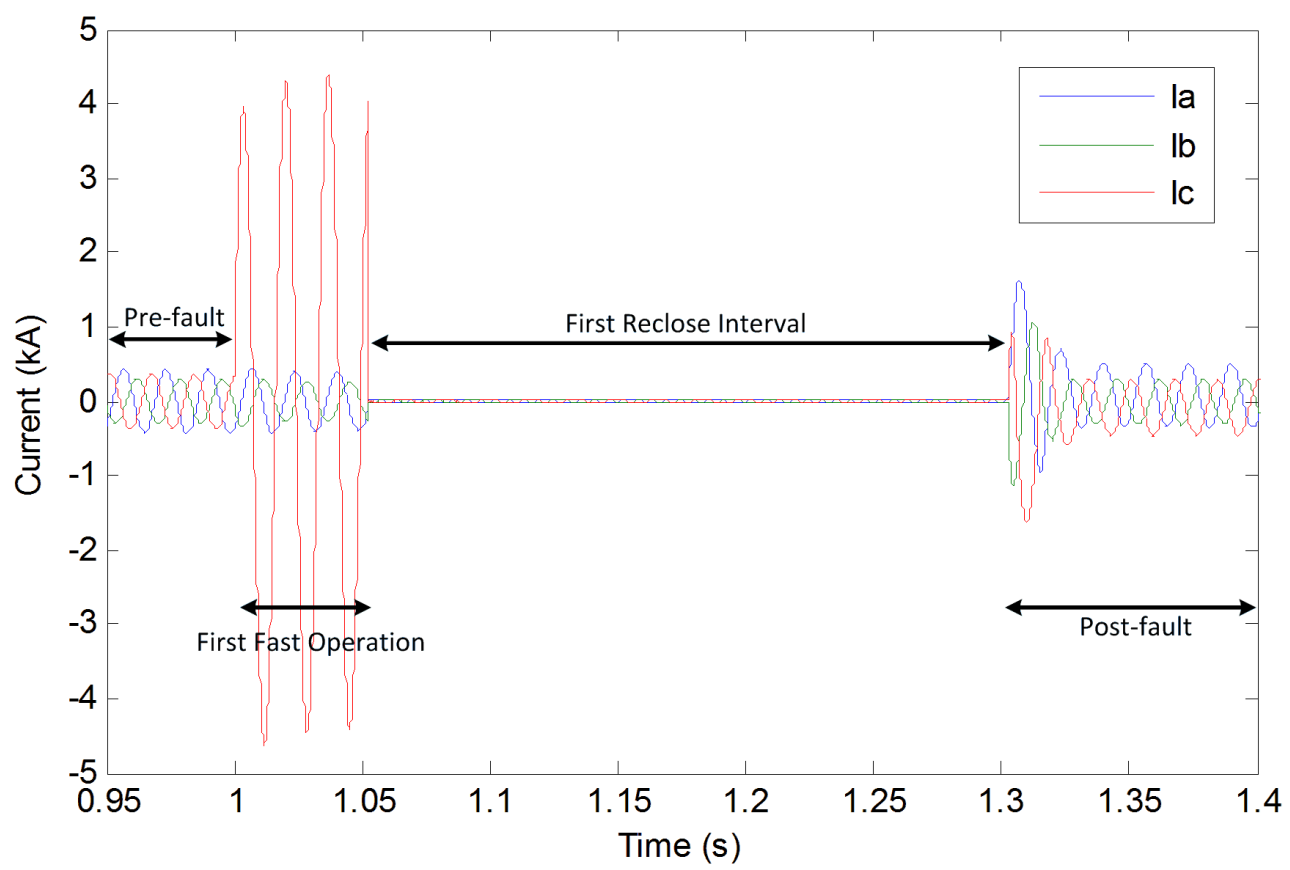

Figure 5.2.2: PSCAD simulation results for one fast operation 


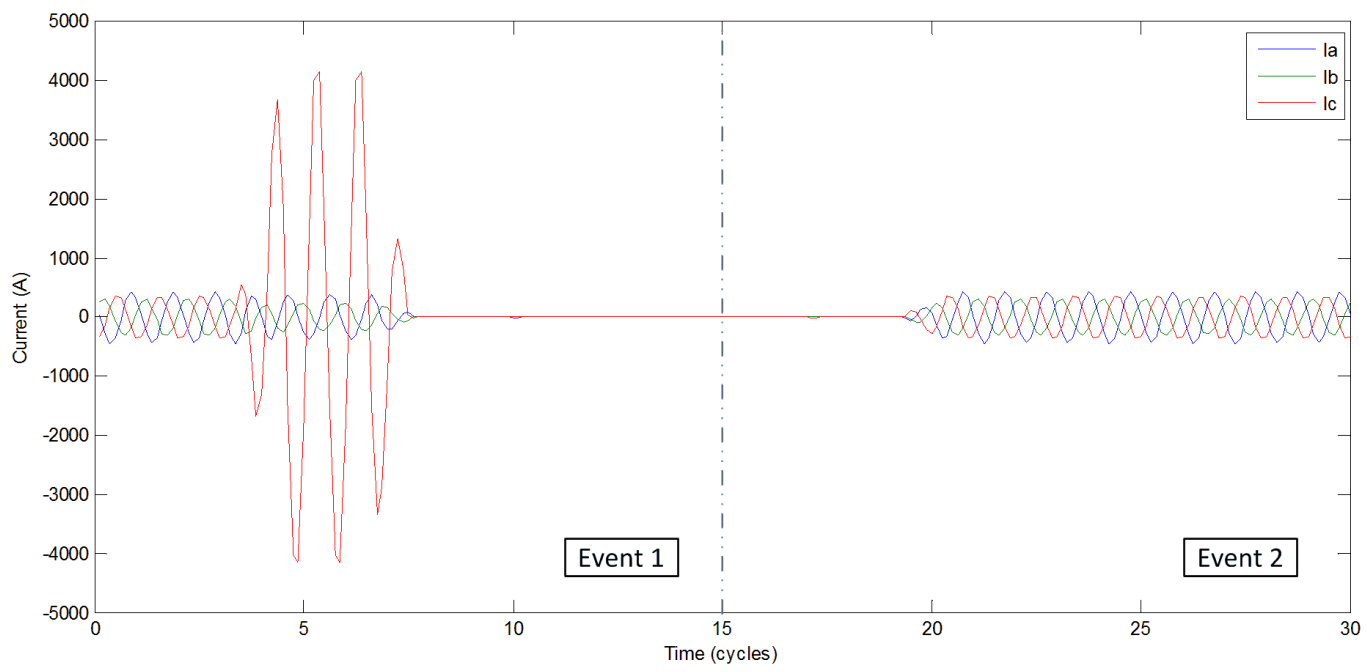

Figure 5.2.3: SEL event recorder data for one fast operation

\subsection{Temporary Fault: Two Fast Operations}

Similar to the first scenario, this scenario simulates a temporary single line-to-ground fault on phase $\mathrm{C}$ on the lateral, downstream of the fuse (see Figure 4.1.1). However, in this case the fault persists longer on the system and the recloser will operate twice on the fast curve. The fault will self-clear after the second fast operation during the second reclose interval. For both the PSCAD and SEL-551c simulations, the expected recloser sequence for two fast operations is shown in Figure 5.3.1. Note that six states are required to implement this fault condition using the SEL test interface. 


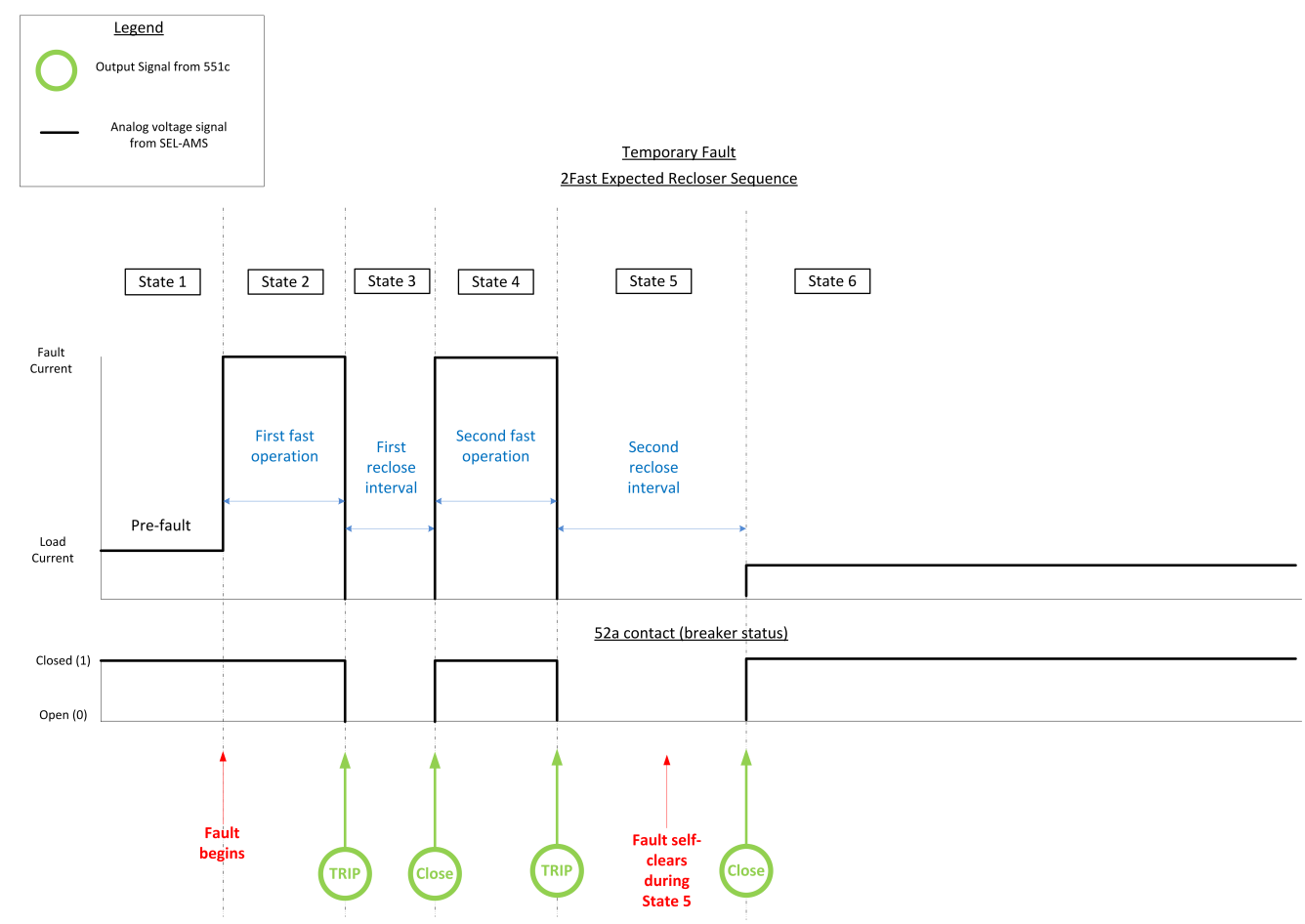

Figure 5.3.1: Expected recloser sequence for two fast operations

Results from the PSCAD simulation are provided below in Figure 5.3.2. We observe that the recloser model operates as expected. Results from the SEL-551c are shown in Figure 5.3.3. Note that three events are captured: two TRIP events and one CLOSE event. As before, we are only able to observe the duration of the fast operations in Events 1 and 2. We are unable to observe the duration of the reclose intervals from the waveform data because it spans two different events. However, because the SEL test interface records when the TRIP and CLOSE signals are asserted by the relay, we can determine the length of the reclose intervals. This analysis of the operating time and reclose intervals for both SEL and PSCAD simulations will be done in Chapter 6 . 


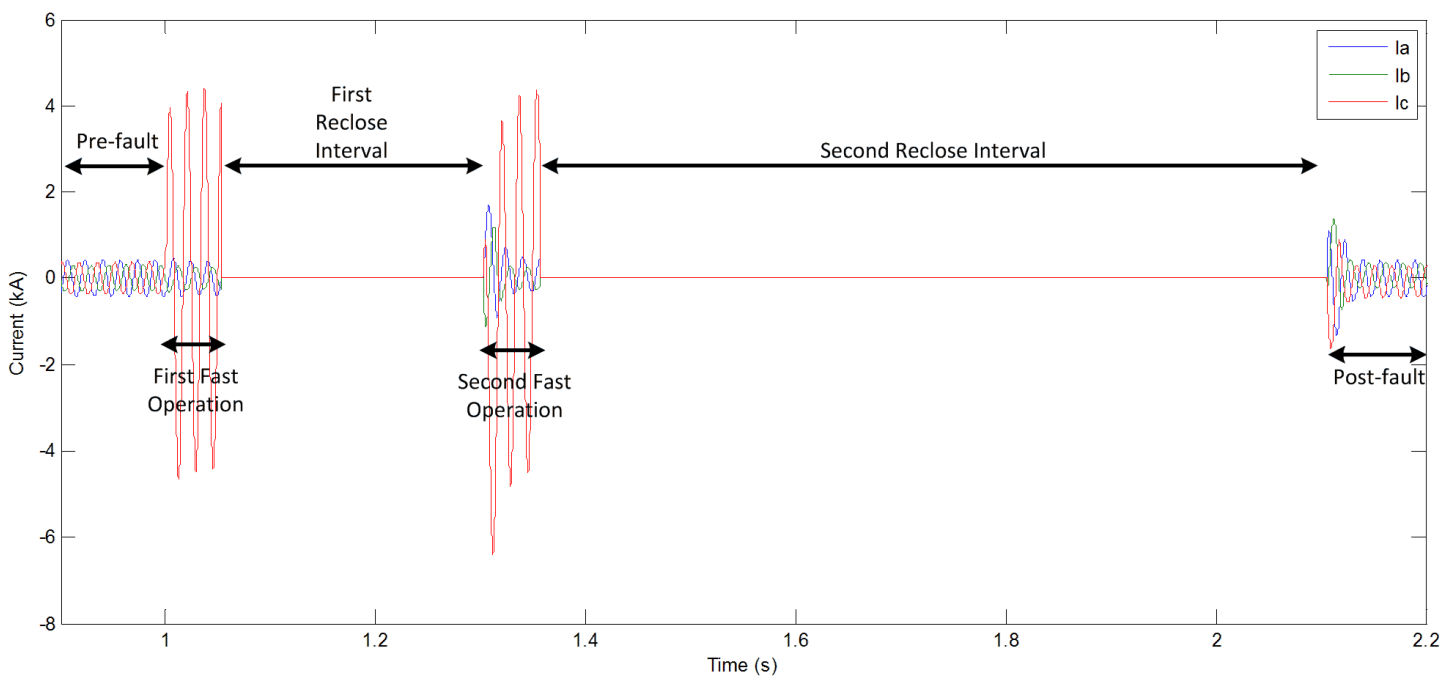

Figure 5.3.2: PSCAD simulation results for two fast operations

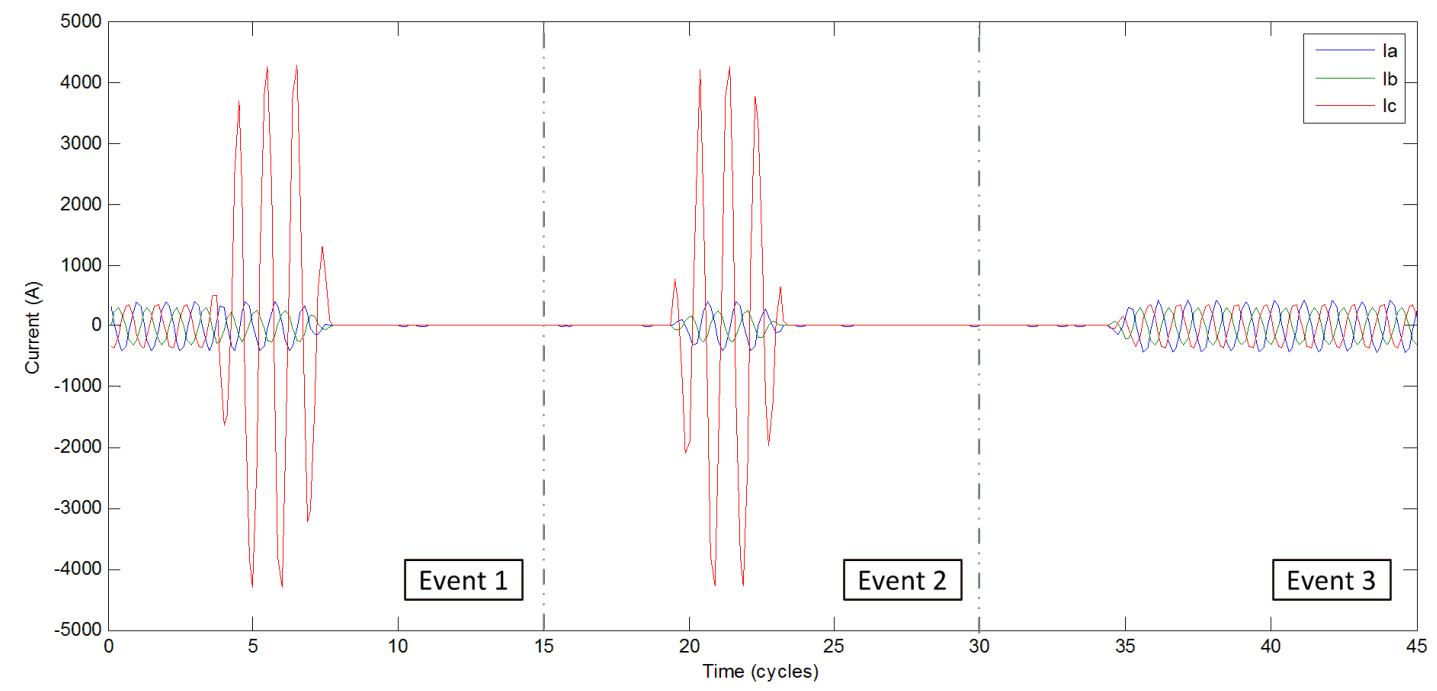

Figure 5.3.3: SEL event recorder data for two fast operations 


\subsection{Permanent Fault: Two Fast Operations, Fuse Clears Fault}

In this scenario, a permanent single line-to-ground fault is simulated on phase $\mathrm{C}$ on the lateral, downstream of the fuse (see Figure 4.1.1). The recloser is set for two fast, two delayed operations, however the fuse clears the fault after the second reclose interval. For both the PSCAD and SEL-551c simulations, the expected recloser sequence for this scenario is shown in Figure 5.4.1. Note that seven states are required to implement this fault condition using the SEL test interface.

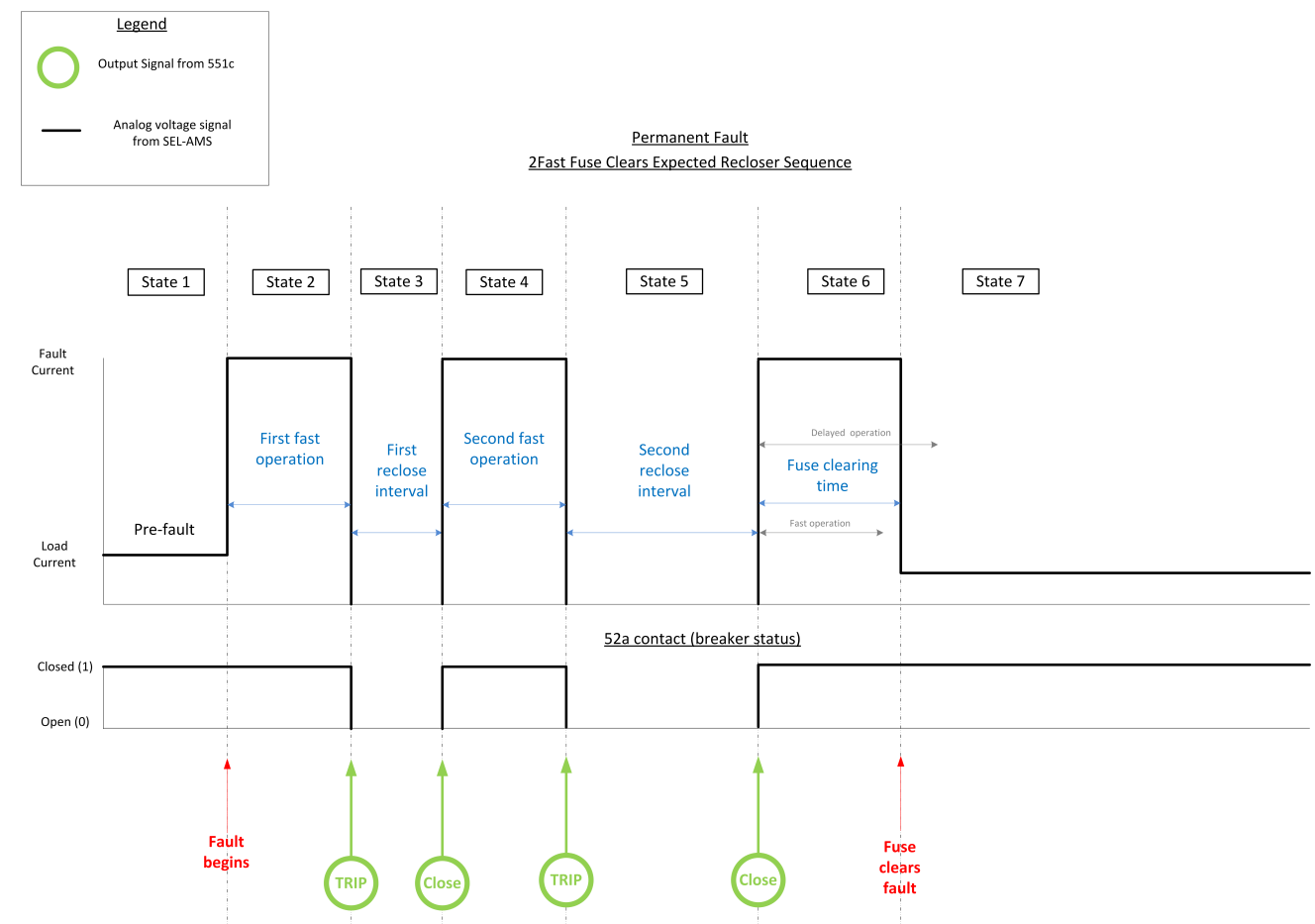

Figure 5.4.1: Expected recloser sequence for permanent fault downstream of fuse

Results from the PSCAD simulation are provided below in Figure 5.4.2. We observe that the recloser model operates as expected. Results from the SEL-551c are shown in Figure 5.4.3. Note that three events are captured: two TRIP events and one CLOSE event. In this case, the fuse clears the fault before the first delay operation times out. Similar to previous scenarios, we are only able to observe the duration of the fast operations in Events 1 and 2 and the fuse operation in Event 3. We are unable to observe the duration of the reclose intervals from the waveform data 
because it spans two events. However, because the SEL test interface records when the TRIP and CLOSE signals are asserted by the relay, we can determine the length of the reclose intervals. This analysis of the operating time and reclose intervals for both SEL and PSCAD simulations will be done in Chapter 6 .

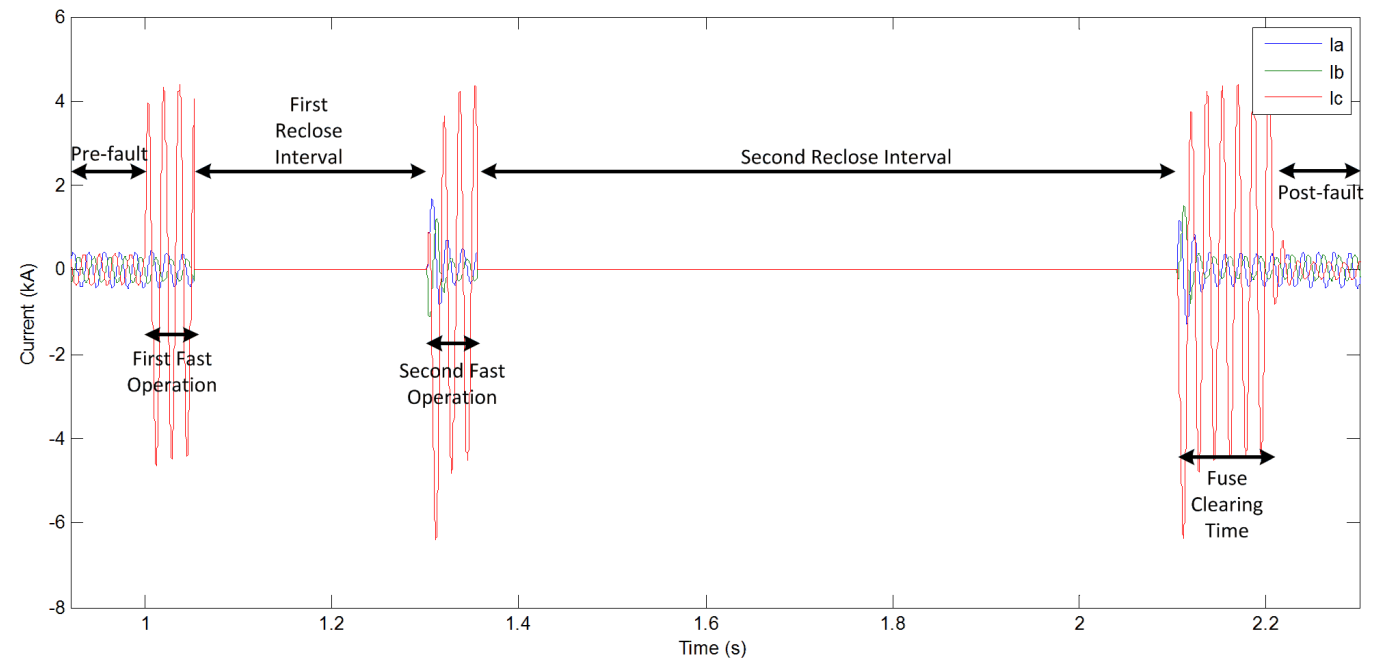

Figure 5.4.2: PSCAD simulation results for permanent fault downstream of fuse

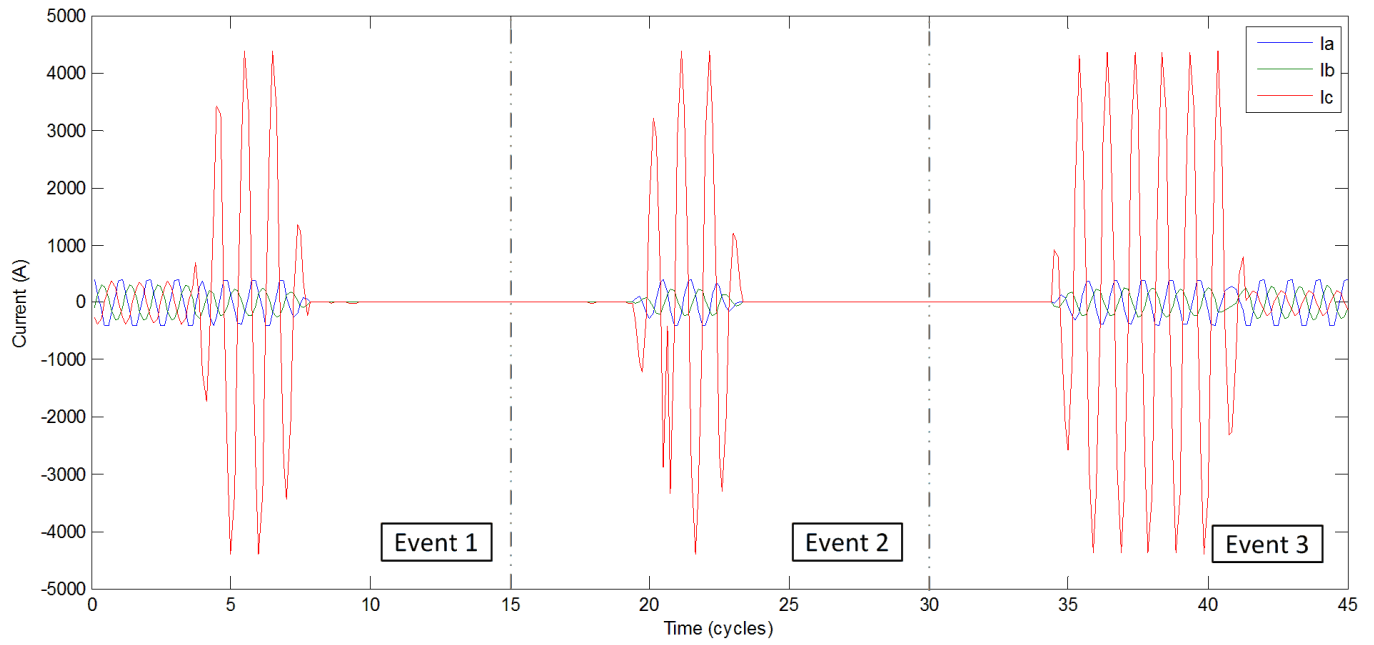

Figure 5.4.3: SEL event recorder data for permanent fault downstream of fuse 


\subsection{Permanent Fault: Two Fast, Two Delayed Op- erations and Recloser Lockout}

In this final scenario, a permanent single line-to-ground fault is simulated on phase $\mathrm{C}$ on the lateral. However, in this case, the fault is located upstream of the fuse (see Figure 4.1.1). Due to the location of the fault, the fuse will not see any fault current and the recloser will operate through its entire reclosing sequence and then lockout. For both the PSCAD and SEL-551c simulations, the expected recloser sequence for this scenario is shown in Figure 5.5.1. Note that nine states are required to implement this fault condition using the SEL test interface.

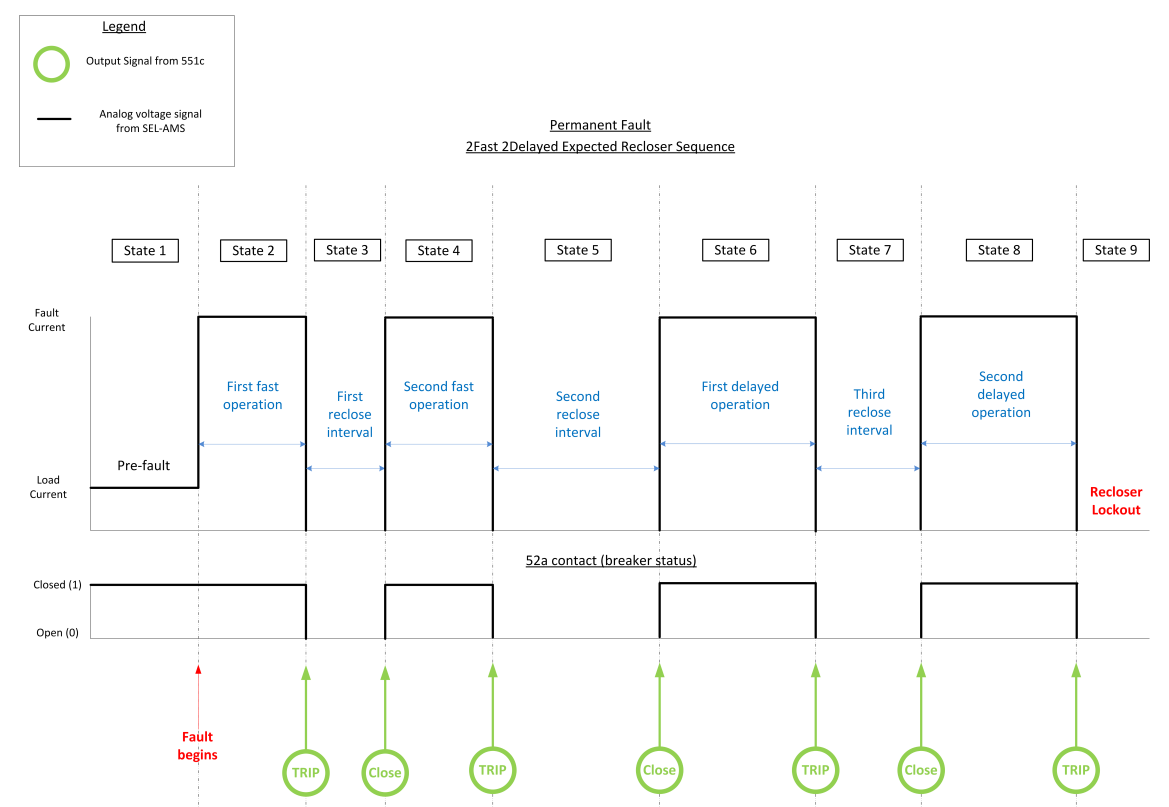

Figure 5.5.1: Expected recloser sequence for permanent fault upstream of fuse

Results from the PSCAD simulation are provided below in Figure 5.5.2. We observe that the recloser model operates as expected. Results from the SEL-551c are shown in Figure 5.5.3. Note that four TRIP events are captured. In this case, because the fault is permanent and the fuse does not see the fault, the recloser locks out at the end of its full sequence. Similar to previous scenarios, we are only able to observe the duration of the time-overcurrent operations in Events 1, 2, 3 and 4 . We 
are unable to observe the duration of the reclose intervals from the waveform data because it spans two events. However, because the SEL test interface records when the TRIP and CLOSE signals are asserted by the relay, we can determine the length of the reclose intervals. This analysis of the operating time and reclose intervals for both SEL and PSCAD simulations will be done in Chapter 6 .

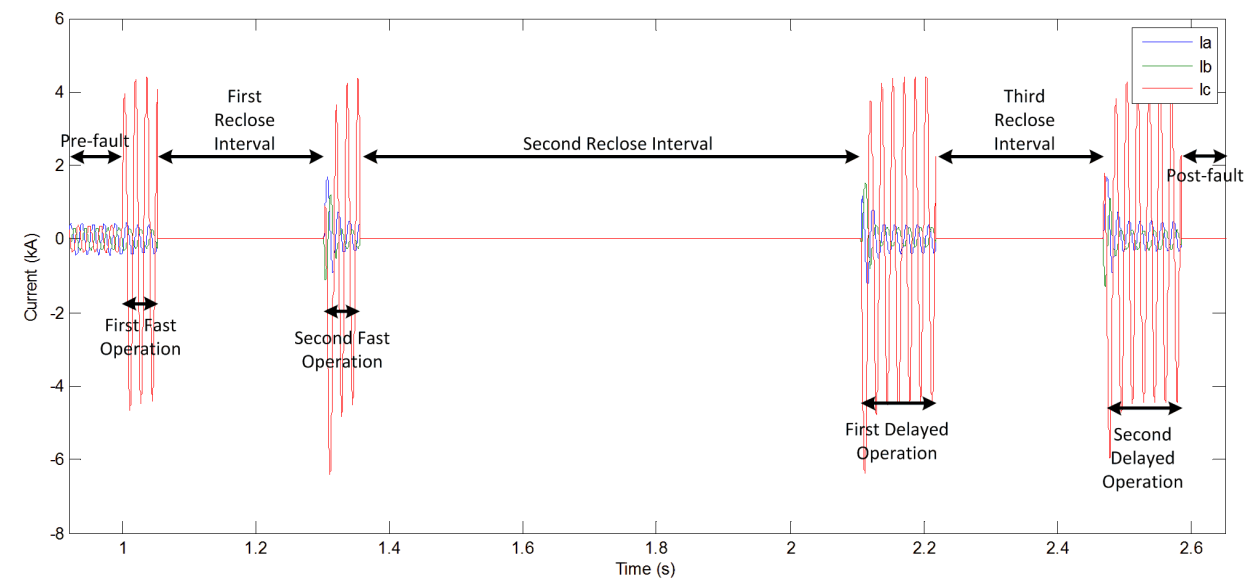

Figure 5.5.2: PSCAD results for permanent fault upstream of fuse

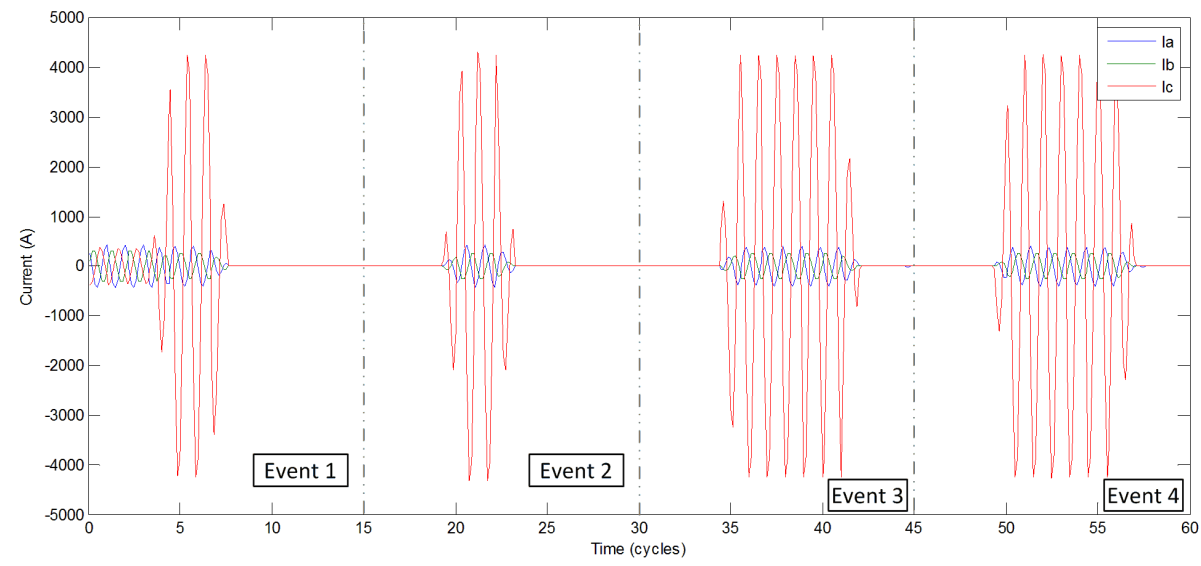

Figure 5.5.3: SEL event recorder data for permanent fault upstream of fuse 


\section{6 \\ Evaluating Performance of Recloser Model}

\subsection{Overview}

In Chapter 5, four fault conditions were simulated in the PSCAD recloser model which resulted in four different reclosing sequences. The fault current waveform was then replicated using the SEL test interface to simulate the same fault conditions on the SEL-551c relay. Resulting waveforms were then extracted from PSCAD and the SEL event recorder and plotted in Matlab. The goal of this chapter is to analyze the waveforms to determine how the PSCAD recloser model and SEL-551c recloser performed when compared to the theoretical values from the TCC settings.

An overview of the data flow is show in Figure 6.1.1. This first step was to model the recloser in PSCAD as discussed in Chapter 4. Next, simulations were run for scenarios discussed in Chapter 5 to obtain the fault current magnitudes and phase angles. These values were then input into the SEL 5401 software to simulate the same fault conditions on the SEL-551c. The SEL test interface, using the replicated fault conditions, was then able to simulate the same faults on the relay. The fault current waveforms and relay performance data was then extracted from the SEL event recorder and converted into a data format (.csv) usable in Matlab. After the data has been imported to Matlab, various types of analysis, such as the wavelet domain analysis in 14 can be completed. However, because the resolution of the SEL event recorder data is limited to 8 samples per cycle, an accurate comparison between PSCAD and event recorder data could not be made in the wavelet domain. The primary analysis done in this work will be comparing the manually calculated 
relay operating times from the PSCAD time-domain simulation with the SEL event recorder waveform and SEL 5401 data.

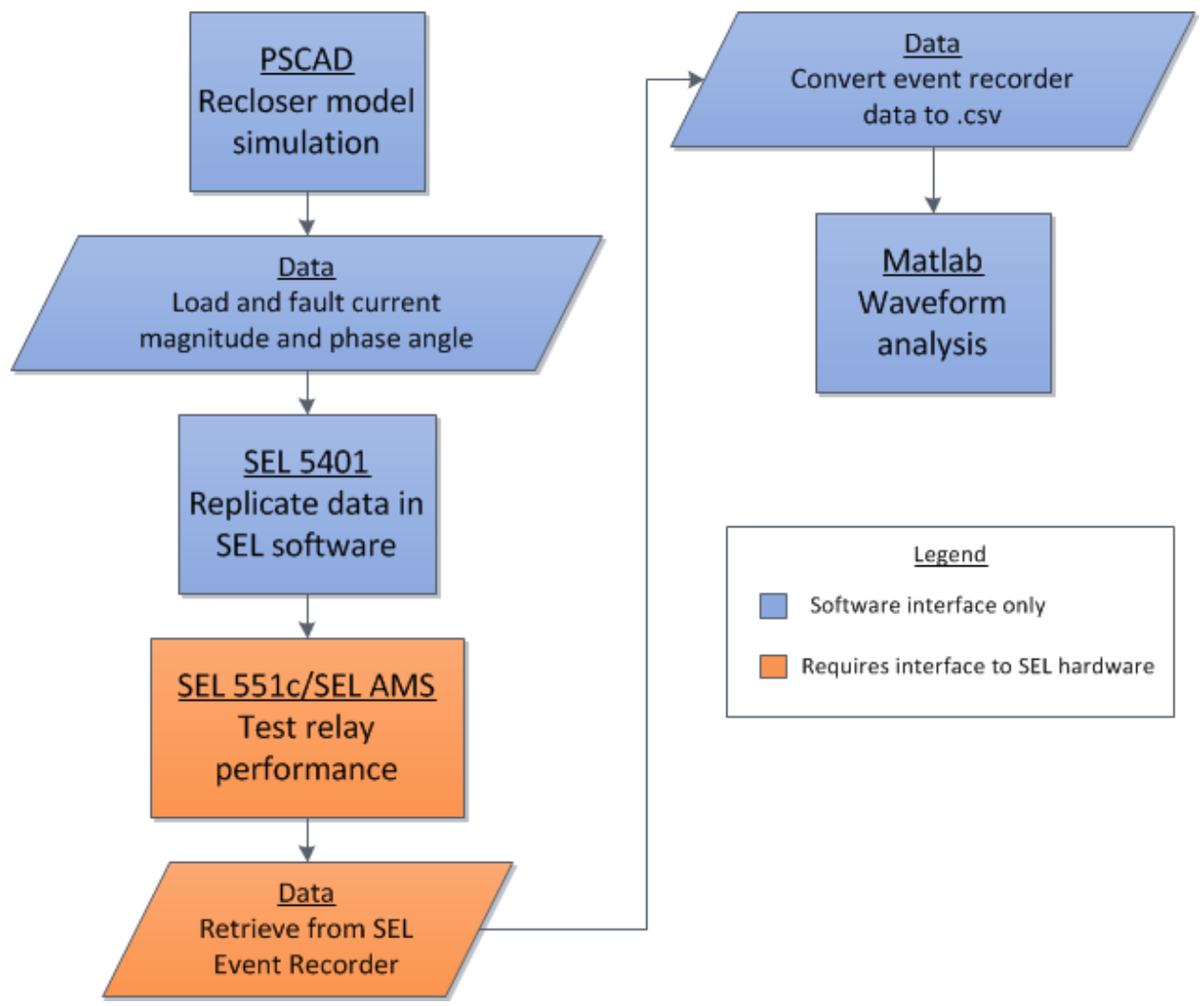

Figure 6.1.1: Overview of data flow

\subsection{Data Analysis}

The data analysis will compare theoretical values from the TCC curves with the PSCAD waveforms and SEL 5401 data. As discussed in Chapter 5, TCC curve type, time delay, and pickup settings are chosen based on the SEL event recorder limitations. These settings are shown in Table 5.2. One can calculate the theoretical operating times for the fast and delayed curves by using the equations in Tables 3.3 and 3.4. For the experiments in Chapter 5, the I.E.C. short-time inverse (C5) curve, Equation 6.2.1, is utilized for both fast and delayed curves in the SEL-551c relay. The time-delay setting is changed to achieve the two operating times

$$
\mathrm{t}=\mathrm{TD} \cdot\left(\frac{0.05}{\mathrm{M}^{0.04}-1}\right)
$$


where TD is the time-delay setting and $\mathrm{M}$ is multiples of pickup. For the PSCAD simulation model, the values in Table 6.1 show the input into the generic TCC equation of the PSCAD time-overcurrent block, Equation 6.2.2. The theoretical operating time is shown in the column $\mathrm{t}_{\mathrm{TCC}}$. The reclose interval settings for both the PSCAD simulation and SEL-551c relay are shown in Table 6.2 .

$$
\mathrm{t}=\mathrm{TD} \cdot\left(\frac{\mathrm{A}}{\mathrm{M}^{\mathrm{p}}-1}+\mathrm{B}\right)+\mathrm{K}
$$

Table 6.1: Theoretical Values From TCC Curves

\begin{tabular}{|c|c|c|c|c|c|c|c|c|c|}
\hline Curve Type & $\mathrm{I}_{\text {fault }}$ & Pickup & $\mathrm{M}$ & $\mathrm{TD}$ & $\mathrm{p}$ & $\mathrm{A}$ & $\mathrm{B}$ & $\mathrm{K}$ & $\mathrm{t}_{\mathrm{TCC}}(\mathrm{s})$ \\
\hline \hline Fast Curve & 4.4 & 1.1 & 4 & 0.05 & 0.04 & 0.05 & 0 & 0 & 0.0438 \\
\hline Slow Curve & 4.4 & 1.1 & 4 & 0.12 & 0.04 & 0.05 & 0 & 0 & 0.1052 \\
\hline
\end{tabular}

Table 6.2: Reclose Interval Settings

\begin{tabular}{|c|c|}
\hline Reclose Interval & Duration (s) \\
\hline \hline 1 & 0.25 \\
\hline 2 & 0.75 \\
\hline 3 & 0.25 \\
\hline
\end{tabular}

The methodology for the PSCAD simulation data is to manually calculate the time-overcurrent operation time from the fault current waveform. This is achieved by observing the duration of the fault and reclose intervals on the faulted phase. For the SEL-551c, event recorder data is used to determine time-overcurrent element operating time. However, because the reclose intervals cannot be accurately observed in the captured waveforms, the duration between TRIP and CLOSE signals from the relay is calculated to determine reclose interval durations. The results for a temporary fault resulting in one fast operation are shown in Tables 6.3 and 6.4 . The column $t_{\text {sim }}$ specifies either the manually calculated or measured times for the simulation model or SEL-551c relay. The column $\Delta t$ specifies the difference between $\mathrm{t}_{\text {sim }}$ and $\mathrm{t}_{\mathrm{TCC}}\left(\Delta t=\mathrm{t}_{\mathrm{sim}}-\mathrm{t}_{\mathrm{TCC}}\right)$. We observe that for the PSCAD simulation, the operating times are relatively close to the expected results with the error being larger for the first fast operation than the reclose interval. For the SEL-551c relay, 
we observe that there is twice as much error for the first fast operation compared to the PSCAD simulation. And for the first reclose interval, there is significant error. In general, the SEL-551c operated close to but not exactly as expected.

Table 6.3: PSCAD Simulation Results For One Fast Operation

\begin{tabular}{|c|c|c|}
\hline Operation & $t_{\text {sim,model }}(\mathrm{s})$ & $\Delta t$ (compared to TCC) \\
\hline \hline Fast 1 & 0.0474 & 0.0036 \\
\hline Reclose Interval 1 & 0.2498 & -0.0002 \\
\hline
\end{tabular}

Table 6.4: SEL-551c Results For One Fast Operation

\begin{tabular}{|c|c|c|}
\hline Operation & $\mathrm{t}_{\text {sim,relay }}(\mathrm{s})$ & $\Delta t$ (compared to TCC) \\
\hline \hline Fast 1 & 0.0510 & 0.0072 \\
\hline Reclose Interval 1 & 0.3953 & 0.1453 \\
\hline
\end{tabular}

The results for a temporary fault resulting in two fast operations are shown in Tables 6.5 and 6.6. The column $t_{\text {sim }}$ specifies either the manually calculated or measured times for the simulation model or SEL-551c relay. The column $\Delta t$ specifies the difference between the simulation and the theoretical values. We observe again that for the PSCAD simulation, the operating times are relatively close to the expected results with the error being largest for the first fast operation. However, the error is significantly reduced by the second fast operation. The first reclose interval has relatively low error, however, error is significantly higher in the second reclose interval.

For the SEL-551c relay, we observe that there is more error for the first fast operation compared to the PSCAD simulation (although less than in the previous scenario). This error is reduced by over $50 \%$ for the second fast operation. Again, as with the previous scenario, there is significant error in the reclose intervals. In general, the SEL-551c operated close to but not exactly as expected. 
Table 6.5: PSCAD Simulation Results for Two Fast Operations

\begin{tabular}{|c|c|c|}
\hline Operation & $t_{\text {sim,model }}(\mathrm{s})$ & $\Delta t$ (compared to TCC) \\
\hline \hline Fast 1 & 0.0474 & 0.0036 \\
\hline Fast 2 & 0.0449 & 0.0011 \\
\hline Reclose Interval 1 & 0.2494 & -0.0006 \\
\hline Reclose Interval 2 & 0.7475 & -0.0025 \\
\hline
\end{tabular}

Table 6.6: SEL-551c Results For Two Fast Operations

\begin{tabular}{|c|c|c|}
\hline Operation & $\mathrm{t}_{\text {sim,relay }}(\mathrm{s})$ & $\Delta t$ (compared to TCC) \\
\hline \hline Fast 1 & 0.0490 & 0.0052 \\
\hline Fast 2 & 0.0459 & 0.0021 \\
\hline Reclose Interval 1 & 0.3953 & 0.1453 \\
\hline Reclose Interval 2 & 0.8958 & 0.1458 \\
\hline
\end{tabular}

The results for a permanent fault downstream of the fuse are shown in Tables 6.7 and 6.8. The column $t_{\text {sim }}$ specifies either the manually calculated or measured times for the simulation model or SEL-551c relay. The column $\Delta t$ specifies the difference between the simulation and the theoretical values. We observe that for the PSCAD simulation, the operating times are relatively close to the expected results. However, in this case, the largest errors are associated with the first fast operation and the fuse operation. As before, the error is reduced for the second fast operation. Similar to previous results, the first reclose interval has relatively low error and the second reclose interval has approximately three times more.

For the SEL-551c relay, we observe that there is high error for the first fast operation. The error in the second fast operation is similar to the previous scenario. Again, we observe that there is significant error in the reclose intervals. It is now apparent that the error in the reclose interval is approximately a constant error of about $0.145 \mathrm{sec}$. In general, the SEL-551c operated close to but not exactly as expected. 
Table 6.7: PSCAD Simulation Results For Permanent Fault Downstream of Fuse

\begin{tabular}{|c|c|c|}
\hline Operation & $\mathrm{t}_{\text {sim,model }}(\mathrm{s})$ & $\Delta t($ compared to TCC) \\
\hline \hline Fast 1 & 0.0476 & 0.0038 \\
\hline Fast 2 & 0.0448 & 0.0010 \\
\hline Fuse 1 & 0.1040 & 0.0040 \\
\hline Reclose Interval 1 & 0.2508 & 0.0008 \\
\hline Reclose Interval 2 & 0.7475 & -0.0025 \\
\hline
\end{tabular}

Table 6.8: SEL-551c Results for Permanent Fault Downstream of Fuse

\begin{tabular}{|c|c|c|}
\hline Operation & $\mathrm{t}_{\text {sim,relay }}(\mathrm{s})$ & $\Delta t($ compared to TCC) \\
\hline \hline Fast 1 & 0.0520 & 0.0082 \\
\hline Fast 2 & 0.0461 & 0.0023 \\
\hline Fuse 1 & 0.1000 & 0.0000 \\
\hline Reclose Interval 1 & 0.3965 & 0.1465 \\
\hline Reclose Interval 2 & 0.8947 & 0.1447 \\
\hline
\end{tabular}

The results for a permanent fault upstream of the fuse are shown in Tables 6.9 and 6.10. The column $t_{\text {sim }}$ specifies either the manually calculated or measured times for the simulation model or SEL-551c relay. The column $\Delta t$ specifies the difference between the simulation and the theoretical values. We observe that for the PSCAD simulation, the operating times are relatively close to the expected results. However, errors are largest for the first fast operation and the second delayed operation. Similar to previous scenarios, the error is reduced for the intermediate operations (second fast and first delayed). The first and second reclose intervals have relatively low error, however, the error for the third reclose interval is relatively high compared to previous results.

For the SEL-551c simulation, we observe that the highest error is with the first fast operation with the second highest error in the second delayed operation. The error in the second fast operation is similar to the previous scenario; this error is close to that of the first delayed operation. Again, we observe that there is significant error in the reclose intervals. However, as noticed in the previous scenario, the error in the reclose intervals appears to be approximately a constant around $0.145 \mathrm{sec}$. In general, the SEL-551c operated close to but not exactly as expected. 
Table 6.9: PSCAD Simulation Results for Permanent Fault Upstream of Fuse

\begin{tabular}{|c|c|c|}
\hline Operation & $\mathrm{t}_{\text {sim,model }}(\mathrm{s})$ & $\Delta t$ (compared to TCC) \\
\hline \hline Fast 1 & 0.0473 & 0.0035 \\
\hline Fast 2 & 0.0449 & 0.0011 \\
\hline Slow 1 & 0.1070 & 0.0018 \\
\hline Slow 2 & 0.1081 & 0.0029 \\
\hline Reclose Interval 1 & 0.2501 & 0.0001 \\
\hline Reclose Interval 2 & 0.7499 & -0.0001 \\
\hline Reclose Interval 3 & 0.1883 & -0.0617 \\
\hline
\end{tabular}

Table 6.10: SEL-551c Results for Permanent Fault Upstream of Fuse

\begin{tabular}{|c|c|c|}
\hline Operation & $\mathrm{t}_{\text {sim,relay }}(\mathrm{s})$ & $\Delta t$ (compared to TCC) \\
\hline \hline Fast 1 & 0.0490 & 0.0052 \\
\hline Fast 2 & 0.0461 & 0.0023 \\
\hline Slow 1 & 0.1081 & 0.0029 \\
\hline Slow 2 & 0.1091 & 0.0039 \\
\hline Reclose Interval 1 & 0.3965 & 0.1465 \\
\hline Reclose Interval 2 & 0.8961 & 0.1461 \\
\hline Reclose Interval 3 & 0.3955 & 0.1455 \\
\hline
\end{tabular}

\subsection{Summary of Analysis}

The following conclusions can be made from the analysis in the previous section. It was observed that the PSCAD model simulations were, in general, more accurate to the desired TCC settings. Although there are errors in the fast and slow operations, these errors were relatively small compared to the SEL-551c. A consistently large error was apparent in the first time-overcurrent operation ranging from 0.0038 to 0.0035 sec. The positive values means that the PSCAD model took longer to operate than the specified TCC curve equation. One possible source of error is the difference in the shape of the fault current during the first fast operation. Observing the waveform in Figure 5.5.2, it is clear the dc-offset is larger for the second, third, and fourth time-overcurrent operations. Because the variable for multiples of pickup, M, 
is not changed between reclose operations in the TCC equation, the larger dc-offset is not accounted for. This would lead to a shorter theoretical operating time. A second observation in the PSCAD model is that the reclose interval times are more accurate than the time-overcurrent operations. This is likely the result of PSCAD model logic. Because the delay between reclose operations is determined only by a preset delay block, there are fewer sources of error, such as differences in dc-offset. Lastly, it can be observed that the PSCAD recloser model provides a good approximation to real world relay behavior. Although the operating times are not exactly the same as the theoretical TCC curve values, the results are close enough to use the recloser model in more complicated systems to simulate recloser performance.

For the results from the SEL-551c, it was generally observed that the operating values were close to but not as accurate as the PSCAD model. It should be noted that all the errors associated with the SEL-551c are positive. This means that the relay operated slower than the TCC curves for all time-overcurrent operations and reclose intervals. For a real world device involving the interaction between many components, it is reasonable to expect that there will be a small delay in operating the TRIP and CLOSE signals. Additionally, because the relay signals are measured by the SEL-AMS signal generator, the additional time delay may be due to the mechanical contact closure required for the TRIP and CLOSE commands. Another observation is that the error associated with each reclose interval appears to be constant around $0.145 \mathrm{sec}$. This would indicate that there is an internal interaction either in the SEL-551c relay or SEL-AMS signal generator that is not accounted for in the PSCAD model or TCC equations. Incorporating this delay into the theoretical calculation would eliminate most of the error. 


\section{7 \\ Conclusion and Future Work}

From the analysis of the results presented, the goal of creating a PSCAD recloser model that emulates the recloser operation of the SEL-551c relay was primarily achieved. An overview of overcurrent protection was provided in Chapter 2. A description of the capabilities of the SEL-551c relay was provided in Chapter 3. The PSCAD recloser model was then developed in Chapter 4. Using this model, a methodology to compare the PSCAD model with the SEL-551c was developed in Chapter 5. Finally, the analysis of the results was provided in Chapter 6. The comparison in performance of the relay model with the SEL-551c and theoretical TCC curve equations suggests that although real world overcurrent relays will have longer operating times, the difference can be negligible. Depending on the needs of the study, these minor errors can be acceptable providing a useful recloser model to include in time-domain simulations. Results also suggest that accuracy can be improved by incorporating delays associated with circuit breaker or contact closures and constant value errors as in the case with the reclose intervals.

Future work would involve testing the model and SEL-551c relay for faults other than single line-to-ground faults. Although not as common, validating the recloser model for all possible fault types would provide a robust model to use in any fault study. A similar comparison and validation can be made using the methodology in this work. Further analysis would involve a more complex system with multiple branches and laterals. In such a system, it would be beneficial to use the information in the reclosing sequence to help with existing fault locating algorithms. For example, if fault waveform data is only available at the substation from a power quality monitoring device, knowing the duration of the time-overcurrent operations or re- 
close interval durations can help identify which protective device has operated. This methodology was initially developed in [14] for single line-to-ground faults, however, building upon the analysis using the PSCAD model developed in this work for other fault types may further help in identifying protective device operations. 


\section{Bibliography}

[1] Pscad/emtdc v. 4.2.

[2] P. M. Anderson. Power System Protection. IEEE Press Power Engineering Series. John Wiley \& Sons, 1999.

[3] J. Bertsch, C. Carnal, D. Karlson, J. McDaniel, and K. Vu. Wide-area protection and power system utilization. Proceedings of the IEEE, 93(5):997 -1003, may 2005.

[4] Lewis J. Blackburn and Thomas J. Domin. Protective Relaying: Principles and Applications. CRC Press, 3rd edition, 2007.

[5] S.M. Brahma and A.A. Girgis. Microprocessor-based reclosing to coordinate fuse and recloser in a system with high penetration of distributed generation. In Power Engineering Society Winter Meeting, 2002. IEEE, volume 1, pages 453-458 vol.1, 2002.

[6] Andres Carvallo and John Cooper. The Advanced Smart Grid: Edge Power Driving Sustainability. Artech House, 2011.

[7] Walter A. Elmore. Protective Relaying Theory and Applications. Marcel Dekker, 2004.

[8] J. Duncan Glover, Mukukutla S. Sarma, and Thomas J. Overbye. Power System Analysis and Design. Thompson, 4th edition, 2008.

[9] Stanley H. Horowitz and Arun G. Phadke. Power System Relaying. John Wiley \& Sons, 3rd edition, 2008.

[10] Chul-Hwan Kim and R. Aggarwal. Wavelet transforms in power systems. i. general introduction to the wavelet transforms. Power Engineering Journal, $14(2): 81-87,2000$.

[11] J. F. Liu, P.L. Wilson, and R.P. Jayasinghe. A pscad/emtdc based simulation study of protective relay. In Developments in Power System Protection, 2004. Eighth IEE International Conference on, volume 1, pages 264-267 Vol.1, 2004. 
[12] Arun G. Phadke and James S. Thorp. Computer Relaying for Power Systems. Research Studies PRess, 1988.

[13] S. Santoso, E.J. Powers, W.M. Grady, and P. Hofmann. Power quality assessment via wavelet transform analysis. Power Delivery, IEEE Transactions on, 11(2):924-930, 1996.

[14] S. Santoso and T.A. Short. Identification of fuse and recloser operations in a radial distribution system. Power Delivery, IEEE Transactions on, 22(4):23702377 , Oct.

[15] Surya Santoso. Fundamentals of Electric Power Quality. Create Space, Winter 2010 edition, 2006.

[16] SEL. SEL-551/SEL-551C Instruction Manual, 2011.

[17] Q.H. Wu, Z. Lu, and T.Y. Ji. Protective Relaying of Power Systems Using Mathematical Morphology. Springer, 2009. 